ARTICLE

https://doi.org/10.1038/s41467-020-14443-5

\title{
Strong and fragile topological Dirac semimetals with higher-order Fermi arcs
}

\author{
Benjamin J. Wieder (1) ${ }^{1 \star}$, Zhijun Wang (10) ${ }^{2,3}$, Jennifer Cano (1) ${ }^{4,5}$, Xi Dai ${ }^{6}$, Leslie M. Schoop (1) ${ }^{7}$, \\ Barry Bradlyn (i) ${ }^{8,9 \star} \&$ B. Andrei Bernevig $1,10,11 \star$
}

Dirac and Weyl semimetals both exhibit arc-like surface states. However, whereas the surface Fermi arcs in Weyl semimetals are topological consequences of the Weyl points themselves, the surface Fermi arcs in Dirac semimetals are not directly related to the bulk Dirac points, raising the question of whether there exists a topological bulk-boundary correspondence for Dirac semimetals. In this work, we discover that strong and fragile topological Dirac semimetals exhibit one-dimensional (1D) higher-order hinge Fermi arcs (HOFAs) as universal, direct consequences of their bulk 3D Dirac points. To predict HOFAs coexisting with topological surface states in solid-state Dirac semimetals, we introduce and layer a spinful model of an $s-d$-hybridized quadrupole insulator (QI). We develop a rigorous nested Jackiw-Rebbi formulation of QIs and HOFA states. Employing ab initio calculations, we demonstrate HOFAs in both the room- $(\alpha)$ and intermediate-temperature $\left(\alpha^{\prime \prime}\right)$ phases of $\mathrm{Cd}_{3} \mathrm{As}_{2}, \mathrm{KMgBi}$, and rutile-structure $\left(\beta^{\prime}-\right) \mathrm{PtO}_{2}$.

\footnotetext{
${ }^{1}$ Department of Physics, Princeton University, Princeton, NJ 08544, USA. ${ }^{2}$ Beijing National Laboratory for Condensed Matter Physics and Institute of Physics, Chinese Academy of Sciences, Beijing 100190, China. ${ }^{3}$ University of Chinese Academy of Sciences, 100049 Beijing, China. ${ }^{4}$ Department of Physics and Astronomy, Stony Brook University, Stony Brook, NY 11974, USA. ${ }^{5}$ Center for Computational Quantum Physics, The Flatiron Institute, New York, NY 10010, USA. ${ }^{6}$ Physics Department, Hong Kong University of Science and Technology, Clear Water Bay, Hong Kong, Hong Kong. ${ }^{7}$ Department of Chemistry, Princeton University, Princeton, NJ 08544, USA. ${ }^{8}$ Department of Physics and Institute for Condensed Matter Theory, University of Illinois at UrbanaChampaign, Urbana, IL 61801-3080, USA. ${ }^{9}$ Donostia International Physics Center, P. Manuel de Lardizabal 4, 20018 Donostia-San Sebastián, Spain. ${ }^{10}$ Dahlem Center for Complex Quantum Systems and Fachbereich Physik, Freie Universität Berlin, Arnimallee 14, 14195 Berlin, Germany. ${ }^{11}$ Max Planck Institute of Microstructure Physics, 06120 Halle, Germany. *email: bwieder@princeton.edu; bbradlyn@illinois.edu; bernevig@princeton.edu
} 
S ince the realization that the Fermi surface of graphene is characterized not only by its bulk 2D Dirac cones, but also by $1 \mathrm{D}$ arc-like states along zigzag edges ${ }^{1}$, there has been an ongoing effort to identify bulk-gapless systems with topological boundary modes. This effort has yielded a wide variety of $3 \mathrm{D}$ nodal semimetals with topological states on their 2D faces, including systems with bulk Weyl ${ }^{2-4}$ and unconventional ${ }^{5-9}$ fermions. Despite the presence of bulk gapless points in these semimetals, bands are still generically gapped in momentum space away from the nodal points, allowing for topological invariants to be defined along closed surfaces in the Brillouin zone $(\mathrm{BZ})^{2,10,11}$. Nontrivial values of these invariants necessitate the presence of topological surface bands. Examples include the surface Fermi arcs in $\mathrm{Weyl}^{2-4}$ and unconventional chiral semimetals $^{6-9,12-15}$, and topological boundary polarization modes, such as the solitons in the Su-Schrieffer-Heeger (SSH) and Rice-Mele chains ${ }^{16,17}$, the aforementioned Fermi arcs in graphene $^{1}$, and the drumhead surface states in centrosymmetric nodal-line semimetals ${ }^{11}$. Researchers have also identified 3D Dirac semimetals with arc-like surface states that resemble the Fermi arcs of Weyl semimetals ${ }^{10,18-20}$. However, unlike the surface states of Weyl, nodal-line, and unconventional chiral semimetals, the surface Fermi arcs in Dirac semimetals can be disconnected and removed without breaking a symmetry or closing a gap $^{21}$, and therefore are not topological consequences of the bulk Dirac points themselves. It has thus remained an open question as to whether 3D Dirac points can actually exhibit robust, nontrivial topology with spectroscopic consequences.

In this work, we exploit the theory of topological quantum chemistry (TQC) ${ }^{22}$ and recent advances in higher-order ${ }^{23-32}$ and fragile $^{33-37}$ topology to discover a large family of 3D Dirac semimetals that exhibit intrinsic, polarization- (quadrupole-) nontrivial higher-order Fermi-arc (HOFA) states on their 1D hinges as direct, topological consequences of their bulk Dirac points, definitively diagnosing condensed matter Dirac fermions as higher-order topological. The HOFA states introduced in this work therefore represent a robust manifestation of a topological bulk-hinge correspondence in experimentally established 3D solid-state semimetals, and may be observable through experimental probes such as scanning tunneling microscopy (STM) and nonlocal quantum oscillation measurements. We support our findings with extensive analytic, tight-binding, and first-principles calculations.

\section{Results}

Boundary modes in topological tuning cycles. To provide context for the analysis performed in this work, we first review the crucial distinctions between topological polarization boundary modes and the surface states of topological insulators (TIs). Whereas in topological (crystalline) insulators 38,39 the Bloch wavefunctions do not admit a description in terms of symmetric, exponentially localized Wannier functions ${ }^{22,40,41}$, insulating phases with only quantized electric polarization conversely do admit a Wannier description; the quantized polarization leads to a nontrivial Berry phase indicating the positions of the electronic Wannier centers relative to the ionic positions ${ }^{22,42}$. In these insulators, such as the SSH chain ${ }^{16,17}$, the Berry phase is quantized by the presence of a crystal symmetry, typically mirror reflection $M$ or spatial inversion $\mathcal{I}$ (Fig. 1b). Correspondingly, the boundary between insulators with differing polarizations forms a domain wall that binds a topological soliton of fractional charge ${ }^{16,17}$, though the energy of this mode may float away from zero if particle-hole symmetry is broken. Nevertheless, as observed in polyacetylene ${ }^{16,43}$, zigzag-terminated graphene ${ }^{44}$, and nodal-line semimetals ${ }^{11,45,46}$, topological polarization boundary modes can still frequently lie near the Fermi energy in real materials.

By reinterpreting one of the momenta as an external parameter, a subset of topological semimetals and (crystalline) insulators, can be reexpressed as the adiabatic, cyclic tuning of an insulator with quantized electric polarization in one fewer dimension $^{40}$. For example, the $y$-directed (hybrid) Wannier centers of a Chern insulator exhibit spectral flow as a function of the momentum $k_{x}$, which can be indicated by the product of parity eigenvalues if $\mathcal{I}$ symmetry is present ${ }^{40,47}$. Reinterpreting $k_{x}$ as an external tuning parameter, we can recast the Chern number, $C$, as a nontrivial tuning cycle (Thouless pump) of a $1 \mathrm{D} \mathrm{SSH}$ chain; as $k_{x}$ is tuned from 0 to $2 \pi$, charge $e C$ is pumped across the unit cell of the crystal. If the cycle is $\mathcal{I}$-symmetric, then $C \bmod 2$ can be detected by (twice) the change in quantized polarization between effective $y$-directed SSH chains at $k_{x}=0$ and $^{17,47} k_{x}=\pi$. We show this schematically in Fig. 1(c). In a crystalline semimetal, the presence of additional symmetries in the tuning cycle can force the gap to close at certain values of $k_{x}$. For instance, adding (spinless) time-reversal $(\mathcal{T})$ symmetry to the $\mathcal{I}$-symmetric Thouless pump obstructs the presence of a nonzero Chern number; in order for the polarization to change by $e / 2$ from $k_{x}=0$ to $k_{x}=\pi$, there must be a gapless point ${ }^{11}$ at some $k_{x}^{*} \in(0, \pi)$, with a time-reversed partner at $-k_{x}^{*}$. A similar gapless point occurs when the polarization of an SSH chain in line group $\mathrm{pm}$ is periodically tuned (Fig. 1d). There, taking the mirror to be $M_{y}$, each value in parameter space indexed by the periodic tuning parameter $k_{x}$ corresponds to a $y$-directed SSH chain with a quantized polarization indicated by the mirror eigenvalues of the occupied bands ${ }^{34}$; each time the polarization jumps between 0 and $\pi$, a robust gapless point forms because the crossing bands carry different mirror eigenvalues.

Recently these arguments were generalized to higher electric multipole moments. In ref. ${ }^{23}$, the authors demonstrated the theoretical existence of spinless insulators with threaded flux that exhibit trivial dipole moments, but topologically quantized electric quadrupole and octupole moments, and which host boundary (corner) modes in two and three dimensions fewer than the bulk, respectively (Fig. 1a, e). Many of these corner-mode phases ${ }^{23,24,26}$ can be identified by their bulk symmetry eigenvalues, exploiting the theory of band representations ${ }^{22,35,36,48-50}$. As shown in recent independent proposals, imposing combinations of rotational, rotoinversion, and $\mathcal{T}$ symmetries allows for 3D topological insulating crystals that are equivalent to nontrivial pumping cycles of quantized quadrupole insulators (QIs) ${ }^{24-27,51}$, or other $2 \mathrm{D}$ phases with corner modes $29,30,32,37,49$. These 3D higher-order TIs ${ }^{24-32}$ host chiral or helical modes not on their $2 \mathrm{D}$ faces, but instead on their $1 \mathrm{D}$ hinges (Fig. 1a, f).

Summary of results. In this work, we present the discovery of higher-order (polarization) topology and HOFA states in a large family of previously identified Dirac semimetals, completing the set of interrelated (higher-order) TIs and semimetals shown in Fig. 1. We demonstrate the intrinsic, topological nature of the HOFA states by performing several extensive calculations that bridge the significant gap between previously established theoretical concepts and the candidate real-material HOFA Dirac semimetals identified in this work. First, we use TQC 22 to formulate a new, spinful model of a QI derived from $s$ - $d$-orbital hybridization in a magnetic layer group, and show that it is topologically equivalent to the spinless model with staggered magnetic flux proposed in ref. ${ }^{23}$ (see Supplementary Note 2). This puts the QI ( $s-d$ hybridization) on the same physical foundation as previous dipole insulators, such as the SSH chain ${ }^{16,17}$ ( $s-p$ hybridization). We then prove using band representations 
a

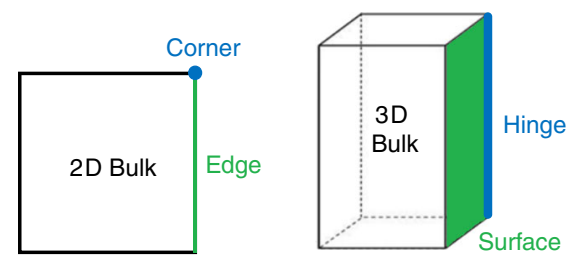

b

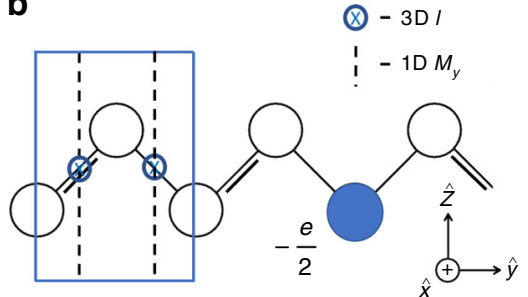

C

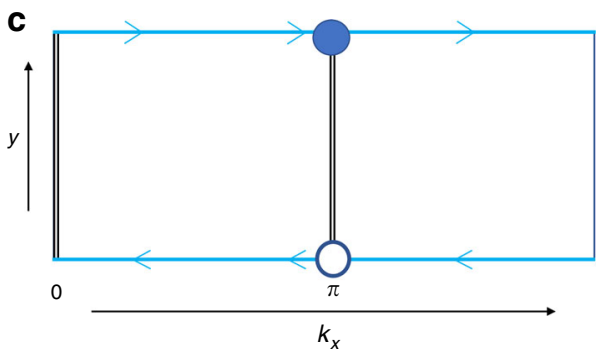

d

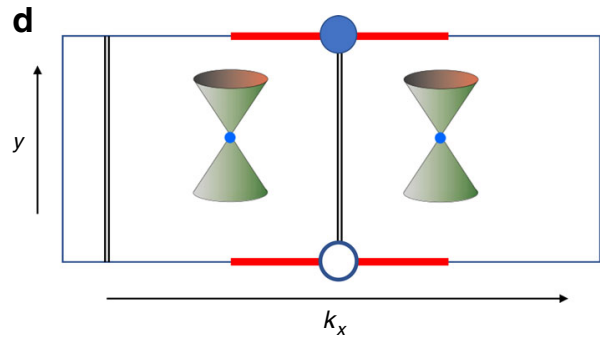

e

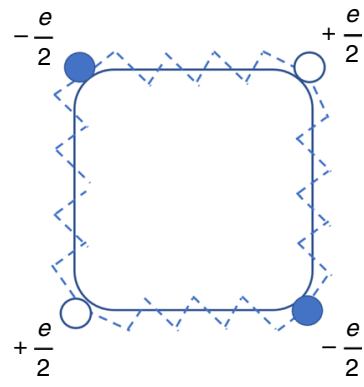

$\mathbf{f}$

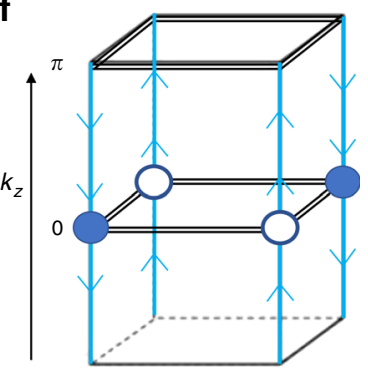

g

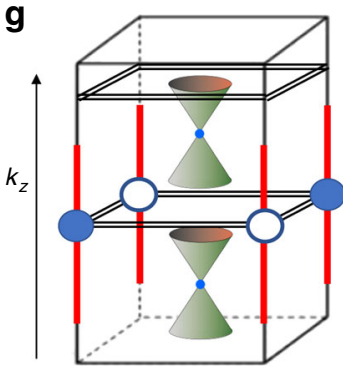

Fig. 1 Tuning cycles of 1D and 2D insulators with OD boundary states. a Terminology for the bulk and boundary of 2D and 3D systems. b A $y$-directed 1D SSH chain with quantized polarization, enforced by either 3D inversion $\mathcal{I}$ or $1 \mathrm{D}$ mirror symmetry along the chain (e.g., the operation $M_{y}$, which takes $y \rightarrow-y)^{16,17}$. c In a $2 \mathrm{D}$ crystal with $\mathcal{I}$ symmetry, $k_{x}$ can be treated as a parameter that periodically tunes between a $y$-directed SSH chain (double black lines in $\mathbf{c}$ and $\mathbf{d}$ ) with zero polarization $\left(k_{x}=0\right)$ and another with $e / 2$ polarization $\left(k_{x}=\pi\right)$, yielding a Chern insulator ${ }^{17,40}$ with chiral edge modes (blue lines). d In a $2 \mathrm{D}$ crystal with $M_{y}$ symmetry instead of $\mathcal{I}$, the Hamiltonian at each value of $k_{x}$ is equivalent to that of a $y$-directed SSH chain with a quantized polarization of $\mathrm{O}$ or e/2; because the polarization cannot change continuously, a periodic tuning cycle indexed by $k_{x}$ between SSH polarizations 0 and e/ 2 must pass through a pair of gapless points. This yields a $2 \mathrm{D}$ band-inverted semimetal with topological polarization modes (red lines) analogous to those in zigzag-terminated graphene ${ }^{1,44}$. e $\mathrm{A} \mathbb{Z}_{2}$ quantized quadrupole insulator $(\mathrm{QI})^{23}$ invariant under wallpaper group p4m. f $\mathrm{A} \mathrm{C}_{4 z}$-broken, mirror-preserving pumping cycle of a QI (double black lines in $\mathbf{f}$ and $\mathbf{g}$ ) is equivalent to a 3D 2 nd-order Chern insulator ${ }^{24-27}$ with chiral hinge modes (blue lines), whereas $\mathbf{g}$ a p4m-preserving cycle is equivalent to a 3D Dirac semimetal with higher-order Fermi arcs (HOFAs) on its 1D hinges (red lines).

that the QI is an obstructed atomic limit with localizable Wannier functions $^{22}$ (Supplementary Note 3 ). Next, we use crystal symmetry to develop an extensive, angular-momentum-based, nested Jackiw-Rebbi ${ }^{52}$ formulation of intrinsic corner modes in order to analytically obtain the bound states of the $s$ - $d$-hybridized QI (Supplementary Note 6) and to relate them to SSH (anti)solitons (Fig. 1(e) and Supplementary Note 6). Because our construction employs an isotropic (i.e., cylindrical) boundary, it uniquely represents an analytic formulation of the QI in which the presence of intrinsic 0D boundary modes can be separated from the extrinsic effects of the singular curvature of sharp corners. Furthermore, because our construction is explicit, general, and rigorous, it can also be employed to predict and analyze other cornermode phases 32,37 . Through our TQC-based model of a QI and our Jackiw-Rebbi analysis, we discover a fragile topological phase ${ }^{33-37}$ that exhibits the same corner charges as a QI modulo $e$; because these charges are a property of the fragile bands closest to the Fermi energy, they persist even when the valence manifold of the fragile phase is trivialized by additional (trivial) bands (Supplementary Notes 4 and 9), as is expected to occur in real materials. Stacking our spinful, TQC-based model of a QI in 3D, we construct both $\mathcal{T}$-symmetric and $\mathcal{T}$-breaking realizations of Dirac semimetals with higher-order Fermi arcs (HOFAs) on their 1D hinges (Fig. 1a, g), i.e., in two fewer dimensions than their bulk. Furthermore, unlike the surface Fermi arcs in Dirac semimetals, which can be removed by symmetry- and bulk-band-orderpreserving potentials ${ }^{21}$, HOFA states represent a direct, topological boundary consequence of the bulk Dirac points.

Crucially, because our analysis is derived from TQC, atomic orbitals, and symmetry-based (nested) Jackiw-Rebbi domain walls, it allows the immediate connection to real materials, unlike recent toy models with HOFA states (i.e., the flux-lattice and particle-hole symmetric semimetallic models in refs. 53,54, respectively) that appeared while we were expanding our material search to fragile and experimentally favorable structural phases of established topological Dirac semimetals. Specifically, while one can naively stack the spinless QI and obtain a toy-model HOFA state, without the careful symmetry- and orbital-based analysis developed in this work, the resulting HOFA states bear no clear connection to 2D TIs (Supplementary Notes 7 and 8), topological crystalline insulator (TCIs) (Supplementary Note 9), fragile phases (Supplementary Notes 4 and 9), or to 3D Dirac points in real materials (Supplementary Notes 12 and 13). Furthermore, in this work, we explicitly relax particle-hole symmetry, which numerous other works, such as ref. ${ }^{54}$, centrally exploit. Because particle-hole is not generically a symmetry of real materials, it can protect corner (and 
thus HOFA) states that appear in toy models, but which are not observable in real materials. This can be understood by making an analogy to the SSH model of polyacetylene ${ }^{16,17}$. Specifically, while real polyacetylene exhibits only $\mathbb{Z}_{2}$ polarization topology 16,17 , the particle-hole symmetric toy-model SSH chain exhibits strong, $\mathbb{Z}$-valued topology (Class AIII in the nomenclature of ref. ${ }^{55}$ ).

We predict previously unidentified HOFAs and related fragilephase corner charges (Supplementary Note 9) in established candidate Dirac semimetals. We present ab initio and tightbinding calculations demonstrating the presence of HOFAs in the intermediate-temperature $\left(\alpha^{\prime \prime}\right)$ phase of the well-studied Dirac semimetal $\mathrm{Cd}_{3} \mathrm{As}_{2}$ in space group (SG) $137\left(P 4_{2} / n m c 1^{\prime}\right)^{10,18,56,57}$ and in the candidate Dirac semimetals $\mathrm{KMgBi}$ in SG 129 $\left(P 4 / n m m 1^{\prime}\right)^{58,59}$ and rutile-structure $\left(\beta^{\prime}-\right) \mathrm{PtO}_{2}$ in SG 136 $\left(P 4_{2} / m n m 1^{\prime}\right)^{60,61}$ (here and throughout this work, we follow ref. ${ }^{62}$ in using primes to denote antiunitary group elements). We also use symmetry arguments to predict that the archetypal room-temperature $(\alpha)$ phase of $\mathrm{Cd}_{3} \mathrm{As}_{2}$ in SG $142\left(I 4_{1} /\right.$ acd $\left.1^{\prime}\right)$ exhibits a related variant of HOFA states that derive from relaxing the reflection symmetries of the QI phase (Supplementary Note 12). Finally, we also demonstrate that, in the presence of an external electric field, the topological Dirac semimetal phase of $\beta^{\prime}-\mathrm{PtO}_{2}$ can be converted into a previously uncharacterized variant of fragile topological Dirac semimetal that displays HOFA states coexisting with fractionally charged corner (hinge) state.

2D TCIs, fragile TIs, and QIs in $\mathbf{p 4 m}$. We begin by providing a more physical formulation of the 2D QI introduced in ref. 23 using atomic orbitals, which clarifies the connection with the SSH chain. We place spin-1/2s and $d_{x^{2}-y^{2}}$ orbitals at the center ( $1 a$ Wyckoff position) of a square unit cell in 2D (Fig. 2(a)) and then, following ref. ${ }^{23}$, impose the symmetries of wallpaper group $p 4 m$, which is generated by $M_{x}$ and $C_{4 z}$ about the $1 a$ position in Fig. 2, as well as $2 \mathrm{D}$ square lattice translations (for the distinctions between wallpaper and layer groups and their relationship to topological semimetals and insulators, see ref. ${ }^{28}$ ). In addition to the symmetries of $p 4 m$, we will first additionally impose $M_{z}$ and $\mathcal{T}$ symmetries to explore $2 \mathrm{D}$ phases with spin-orbit coupling (SOC), and then subsequently relax $M_{z}$ and $\mathcal{T}$ with magnetism to induce the QI. Eliminating all nonessential symmetries and degeneracies, we form the Hamiltonian:

$$
\begin{aligned}
\mathcal{H}(\mathbf{k})= & t_{1} \tau^{z}\left[\cos \left(k_{x}\right)+\cos \left(k_{y}\right)\right]+t_{2} \tau^{x}\left[\cos \left(k_{x}\right)-\cos \left(k_{y}\right)\right] \\
& +v_{m} \tau^{z}+t_{P H} \mathbb{1}_{\tau \sigma}\left[\cos \left(k_{x}\right)+\cos \left(k_{y}\right)\right] \\
& +v_{s} \tau^{y} \sigma^{z} \sin \left(k_{x}\right) \sin \left(k_{y}\right)
\end{aligned}
$$

where $\tau(\sigma)$ indexes the $s, d$-orbital (spin) degree of freedom and $\mathbb{1}_{\tau \sigma}$ is the $4 \times 4$ identity. Here, $v_{m}$ produces on-site orbital splitting, $t_{1}\left(t_{2}\right)$ is first-neighbor hopping between the same (opposite) orbital, $t_{P H}$ is spin- and orbital-independent first-neighbor hopping that explicitly breaks particle-hole symmetry, and $v_{s}$ represents second-neighbor SOC (Supplementary Note 1). Equation (1) is invariant under the symmetries of layer group $p 4 / \mathrm{mmml}^{\prime}$ (Table 1). Since $\mathcal{I}=M_{x} M_{y} M_{z}$ is given by the identity matrix and $\left\{M_{x}, M_{y}\right\}=0$ in the representation in Table 1 , our model with four spinful orbitals (Eq. (1)) exhibits the same bulk symmetry eigenvalues and symmetry algebra as the original, spinless QI model in ref. ${ }^{23}$. The bulk bands of Eq. (1), due to the presence of $\mathcal{I} \times \mathcal{T}$ symmetry, are twofold degenerate (Fig. 2b, c). In Supplementary Note 5 and 6 , we additionally introduce and analyze models of QIs and HOFA semimetals with $p-d$ hybridization.

To diagnose the topology of Eq. (1), we examine the $x$ directed Wilson loop (holonomy) matrix ${ }^{34,36,63}$, a bulk quantity defined by:

$$
\mathcal{W}_{\left(k_{x 0}, k_{y}\right)} \equiv P e^{i \int_{k_{x 0}}^{k_{x 0}+2 \pi} d k_{x} A_{x}\left(k_{x}, k_{y}\right)}
$$

where $P$ indicates that the integral is path-ordered and $A_{x}(k)_{i j} \equiv$ $i\left\langle u^{i}(k) \mid \partial_{k_{x}} u^{j}(k)\right\rangle$ is the matrix-valued Berry connection. The eigenvalues $\theta\left(k_{y}\right)$ of $\mathcal{W}$ are gauge invariant and form bands in one fewer dimension than that of the bulk, with connectivity and degeneracy constrained by the symmetries of the $x$ projected edge symmetry group ${ }^{28}$, as well as by the representations of bulk symmetries ${ }^{34}$. At half filling, Eq. (1) exhibits two topologically distinct insulating phases (Fig. 2c, f), indicated by the relative ordering of the Kramers pairs of $C_{4 z}$ eigenvalues of the occupied bands at $\Gamma$ and $M$ (Fig. 2b) ${ }^{23}$. In Fig. 2, we show the Wilson spectra computed from the lower two bands of Eq. (1) in the uninverted (trivial) phase (e) and in the inverted (nontrivial) phase (h). As we will detail below, we then also calculate the Wilson spectrum of Eq. (1) in the presence of potentials that break $M_{z}$ symmetry while either preserving (k) or breaking (n) $\mathcal{T}$ symmetry; we also compare the Wilson loop spectra to the surface states of tight-binding Hamiltonians calculated in a ribbon geometry $(\mathrm{d}, \mathrm{g}, \mathrm{j}, \mathrm{m})$.

Using Eq. (2), we identify the nontrivial phase of Eq. (1) as a TCI $28,64,65$ with mirror Chern number $C_{M_{z}}=2$ (Fig. $2 \mathrm{~g}, \mathrm{~h}$ ). By introducing a term that breaks $M_{z}$ and $\mathcal{I}^{z}$ while preserving the symmetries of wallpaper group $p 4 m 1^{\prime}$ (generated by $M_{x, y}, C_{4 z}$, and $\mathcal{T})^{28,62}$

$$
V_{M_{z}}(\mathbf{k})=v_{M_{z}}\left[\tau^{z} \sigma^{y} \sin \left(k_{x}\right)-\tau^{z} \sigma^{x} \sin \left(k_{y}\right)\right],
$$

we can gap the edge states of this TCI (Fig. 2j). However, its twoband $x$-directed Wilson loop still winds (Fig. 2k). This phenomenon is related to recently identified fragile topological phases $^{33-37}$, whose Wilson loops can be rendered topologically trivial by the introduction of trivial bands. In Supplementary Note 4, we show how the topological Wilson connectivity of this four-band model is unstable to the addition of spinful $s$ orbitals at the $2 c$ position of $p 4 m 1^{\prime}$. In both the TCI (Eq. (1)) and fragile (Eqs. (1) and (3)) phases, $\mathcal{T}$ symmetry obstructs the presence of singly degenerate corner modes; however, we found that the $M_{z^{-}}$ broken fragile phase, when the overall system is kept at a constant half filling, still exhibits three-quarters-filled Kramers pairs of corner modes that can float into the bulk gap (Supplementary Notes 4 and 9). We specifically show in Supplementary Note 9 that, as this fragile phase can be connected to a QI by restoring $\mathcal{T}$ symmetry without closing a bulk or edge gap, its corner modes still exhibit the same charges as the QI modulo e. $C_{M_{z}}=2$ TCI phases in layer group $p 4 / m m m 1^{\prime}$ have been proposed in $\mathrm{XY}$ $(\mathrm{X}=\mathrm{Sn}, \mathrm{Te} ; \mathrm{Y}=\mathrm{S}, \mathrm{Se}, \mathrm{Te})$ monolayers ${ }^{66}$. However because band inversion in these $\mathrm{XY}$ monolayers occurs at the $X$ and $X^{\prime}$ points (Fig. 2b) between bands with different $\mathcal{I}$ eigenvalues, rather than at the $\Gamma$ or $M$ points between bands with different pairs of $C_{4 z}$ eigenvalues, $\mathrm{XY}$ monolayers will realize a different insulating phase than the fragile phase of Eqs. (1) and (3) when $M_{z}$ is broken with a substrate or an external field (Supplementary Note 9).

To induce the QI phase (Fig. 2l), we first set $v_{M_{z}}=0$ in Eq. (3); this restores $M_{z}$ and $\mathcal{I}$ symmetries. We then instead add to Eq. (1) a term that anticommutes with $\mathcal{H}(\mathbf{k})$ in its particle-hole symmetric limit $\left(t_{P H} \rightarrow 0\right)$ :

$$
U(\mathbf{k})=u\left[\tau^{y} \sigma^{y} \sin \left(k_{x}\right)+\tau^{y} \sigma^{x} \sin \left(k_{y}\right)\right] .
$$

Equation (4) breaks $M_{z}, \mathcal{I}$, and $\mathcal{T}$ while preserving the unitary symmetries of $p 4 m$ and the magnetic antiunitary symmetries $M_{z} \times \mathcal{T}$ and $\mathcal{I} \times \mathcal{T}$, the latter of which continues to enforce a twofold band degeneracy (Fig. 2l). The new Hamiltonian 
a

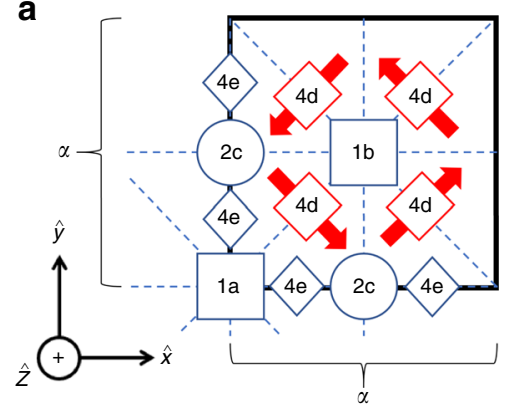

b

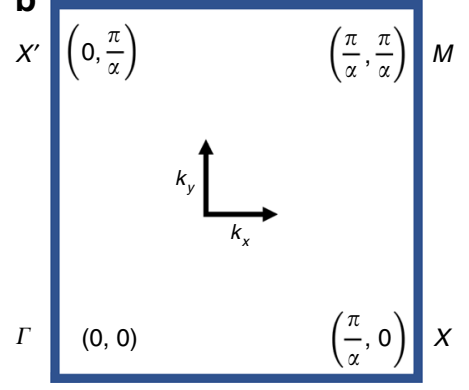

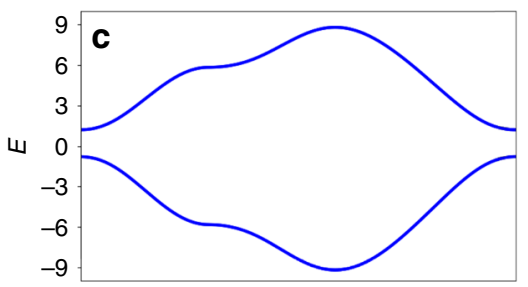

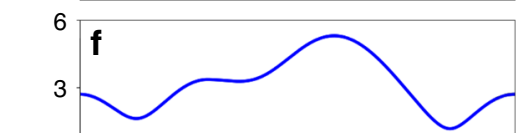

w

$\omega$

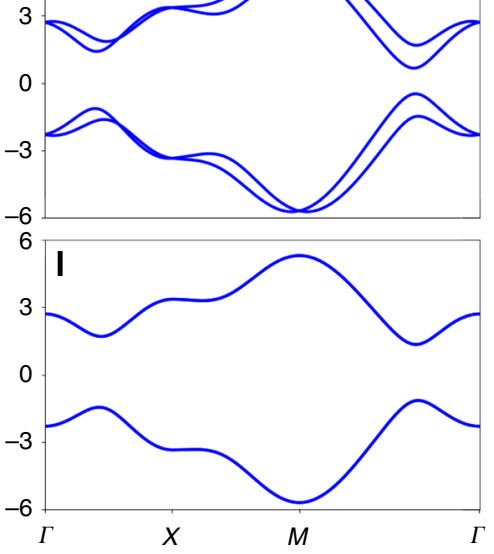

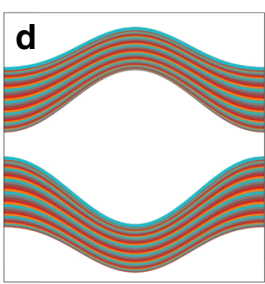
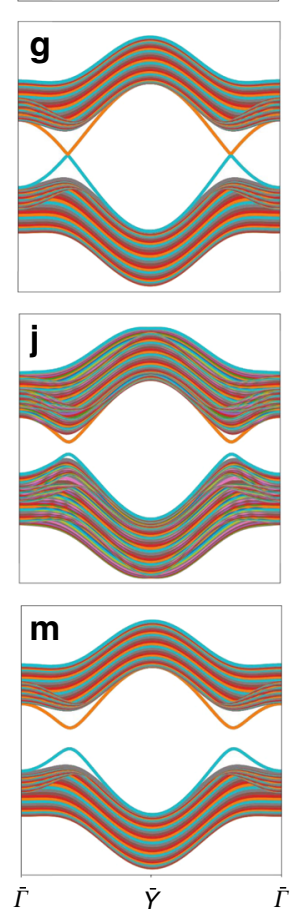

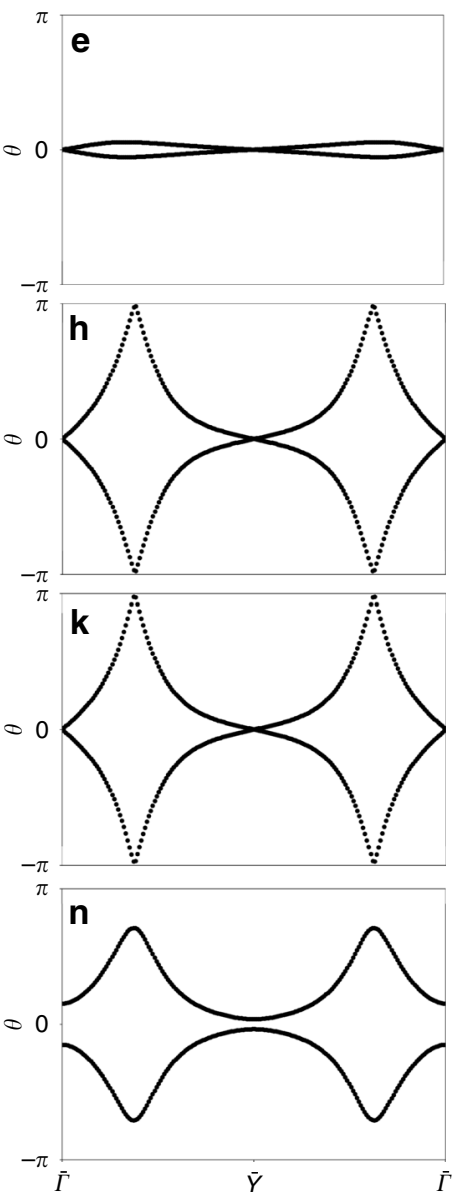

Fig. 2 TCl, fragile, and QI phases in 2D insulators with $\mathbf{p 4 m}$ symmetry. a $\mathcal{T}$-symmetric layer group $p 4 / \mathrm{mmm}^{\prime}$ reduces to type-III magnetic layer group 62 $\mathrm{p} 4 / \mathrm{m}^{\prime} \mathrm{mm}$ under the application of a magnetic potential with no net magnetic moment in each unit cell (Supplementary Note 3); this can be achieved by placing spin-1/2 magnetic moments (red arrows in a) at the $4 d$ Wyckoff position with orientations related by $C_{4 z}$ and $M_{x, y}$. $\mathbf{b}$ BZ and $\mathbf{c}$ bulk bands for a model (Eq. (1)) that respects $p 4 / \mathrm{mmml}^{\prime}$ (Table 1), which has $M_{z}$ and $\mathcal{T}$ symmetries, as well as the symmetries of $p 4 / \mathrm{m}^{\prime} \mathrm{mm}$ in $\mathbf{a}$; this model is constructed from $s$ and $d_{x^{2}-y^{2}}$ orbitals at the 1a Wyckoff position. Eq. (1) can be tuned between a trivial and a mirror TCl phase (f), distinguished by their (d, $\mathbf{g}$ ) ribbon edge spectra and $(\mathbf{e}, \mathbf{h}) x$-directed Wilson loops plotted as functions of $k_{y}$ (Eq. (2)). i Relaxing $M_{z}$ while preserving $C_{2 z}$ and $\mathcal{T}$ by introducing Eq. (3) to Eq. (1), (k) we realize a four-band model with the same Wilson loop winding as the 2D TCI phase in $\mathbf{f}-\mathbf{h}$, but (j) without topological edge states. The Wilson loop in $\mathbf{k}$ can either be trivialized by the addition of more orbitals to the model (Supplementary Note 4), or gapped by the magnetic potential depicted in $\mathbf{a}$. I Upon gapping ( $\mathbf{m})$ the surface and (n) Wilson bands with magnetism that breaks $M_{z}$ while preserving magnetic wallpaper group ${ }^{28,62} p 4 m$, which we accomplish by adding the potential in Eq. (4), the Wannier centers of the topological phases in f-k localize at the $1 b$ position (Supplementary Note 3), realizing an insulator topologically equivalent to the QI introduced in ref. 23 (Supplementary Note 2).

$\mathcal{H}(\mathbf{k})+U(\mathbf{k})$ (Eqs. (1) and (4)) therefore has the symmetry of magnetic layer group $p 4 / \mathrm{m}^{\prime} \mathrm{mm}$, a supergroup of $p 4 \mathrm{~m}$. We note that because $U(\mathbf{k})$ preserves two orthogonal mirrors, $M_{x, y}$, it cannot be induced by a constant Zeeman field alone, and must instead come from several internal magnetic moments or applied quadrupolar magnetism. An example of a configuration of spin$1 / 2$ magnetic moments is shown in Fig. 2a, that like $U(\mathbf{k})$, lowers the symmetry of $p 4 / \mathrm{mmml}^{\prime}$ to $p 4 / \mathrm{m}^{\prime} \mathrm{mm}$. When Eq. (4) is added to Eq. (1), the surface states and Wilson spectrum gap (Fig. $2 \mathrm{~m}$, $\mathrm{n})$, but gapped, $\mathrm{SSH}$-like states remain bound to the $1 \mathrm{D}$ edges $^{23,24}$ (Supplementary Note 11). By projecting onto one of the eigenstates of $\mathcal{W}$ (for example the lower Wilson band in Fig. 2n), a second, nested Wilson loop can be computed in the $y$ direction, and displays a nested Berry phase $\theta_{2}$ of $0(\pi)$ if this magnetic 


\begin{tabular}{|c|c|}
\hline \multicolumn{2}{|c|}{ Symmetries of 2D Hamiltonians $\mathcal{H}\left(\boldsymbol{k}_{x}, \boldsymbol{k}_{\mathbf{y}}\right)$} \\
\hline $\begin{array}{l}g \\
M_{x} \\
M_{y} \\
C_{4 z} \\
M_{z} \\
\mathcal{I} \\
\mathcal{T}\end{array}$ & $\begin{array}{l}g \mathcal{H}\left(g k_{x}, g k_{y}\right) g^{-1} \\
\sigma^{x} \mathcal{H}\left(-k_{x}, k_{y}\right) \sigma^{x} \\
\sigma^{y} \mathcal{H}\left(k_{x},-k_{y}\right) \sigma^{y} \\
\tau^{z}\left(\frac{1_{\sigma}-1 \sigma^{z}}{\sqrt{2}}\right) \mathcal{H}\left(k_{y},-k_{x}\right) \tau^{z}\left(\frac{1_{\sigma}+i \sigma^{z}}{\sqrt{2}}\right) \\
\sigma^{z} \mathcal{H}\left(k_{x}, k_{y}\right) \sigma^{z} \\
\mathcal{H}\left(-k_{x},-k_{y}\right) \\
\sigma^{y} \mathcal{H}^{*}\left(-k_{x},-k_{y}\right) \sigma^{y}\end{array}$ \\
\hline \multicolumn{2}{|c|}{ Symmetries of 3D Hamiltonians $\mathcal{H}\left(k_{x}, k_{y}, k_{z}\right)$} \\
\hline $\begin{array}{l}g \\
M_{x} \\
M_{y} \\
C_{4 z} \\
M_{z} \\
\mathcal{I} \\
\mathcal{T}\end{array}$ & $\begin{array}{l}g \mathcal{H}\left(g k_{x}, g k_{y}, g k_{z}\right) g^{-1} \\
\sigma^{x} \mathcal{H}\left(-k_{x}, k_{y}, k_{z}\right) \sigma^{x} \\
\sigma^{y} \mathcal{H}\left(k_{x},-k_{y}, k_{z}\right) \sigma^{y} \\
\tau^{z}\left(\frac{1_{\sigma}-i \sigma^{2}}{\sqrt{2}}\right) \mathcal{H}\left(k_{y},-k_{x}, k_{z}\right) \tau^{z}\left(\frac{1_{\sigma}+i \sigma^{z}}{\sqrt{2}}\right) \\
\sigma^{z} \mathcal{H}\left(k_{x}, k_{y},-k_{z}\right) \sigma^{z} \\
\mathcal{H}\left(-k_{x},-k_{y},-k_{z}\right) \\
\sigma^{y} \mathcal{H}^{*}\left(-k_{x},-k_{y},-k_{z}\right) \sigma^{y}\end{array}$ \\
\hline
\end{tabular}

The models in Eqs. (3-6) derive from Eq. (1), which contains the symmetries (wallpaper group 28 $p 4 m$ ) of a $\mathrm{Q}^{23,24}$ (Supplementary Note 3 ), as well as $M_{z}, \mathcal{I}=M_{x} M_{y} M_{z}$ and $\mathcal{T}$.

insulator is in a trivial (quadrupole) phase $\mathrm{e}^{23}$. For all nonzero values of $u$ in Eq. (4), transitions between QI and trivial phases occur when the bulk gap closes at $\Gamma(M)$ for $2 t_{1}=-(+) v_{m}$, with $\left|\frac{v_{m}}{t_{1}}\right|<(>) 2$ characterizing the QI (trivial) phase. As we show in Supplementary Note 2, the Hamiltonian $\mathcal{H}(\mathbf{k})+U(\mathbf{k})$ (Eqs. (1) and (4)) is topologically equivalent to the quadrupole model introduced in ref. ${ }^{23}$. We can also choose to reintroduce $V_{M_{z}}(\mathbf{k})$ (Eq. (3)) to Eqs. (1) and (4), which, as it is invariant under $p 4 m$, will preserve the QI phase if it does not close a bulk or edge gap, even though it breaks the combined magnetic symmetries $M_{z} \times \mathcal{T}$ and $\mathcal{I} \times \mathcal{T}$ in $p 4 / \mathrm{m}^{\prime} \mathrm{mm}$, the (magnetic) layer group of Eqs. (1) and (4). For weak $v_{M_{z}}$ this therefore results in a QI phase in $p 4 m$ with singly degenerate bands, and for stronger values, it can induce a crystalline semimetallic phase (Supplementary Note 1). It also follows from the theory of band representations ${ }^{22,35,36}$ that the QI phase of Eqs. (1) and (4) with (without) Eq. (3) is an obstructed atomic limit ${ }^{22}$ with the two occupied Wannier orbitals shifted to the $1 b$ Wyckoff position of $p 4 m\left(p 4 / \mathrm{m}^{\prime} \mathrm{mm}\right)$ (Supplementary Note 3).

In Supplementary Notes 6-10, we construct a microscopic picture of the phase transitions between the TCI, fragile, and QI phases of the tight-binding Hamiltonians given by Eq. (1) with the potentials in Eqs. (3) and (4). We also analytically examine the phase transition between a tight-binding model of a $p_{z}-d_{x^{2}-y^{2}}$-hybridized 2D TI (Supplementary Note 5) and an additional model of QI in $p 4 / \mathrm{m}^{\prime} \mathrm{mm}$ that is distinct from (but topologically equivalent to) Eqs. (1) and (4). Specifically in Supplementary Notes 7 and 8 , we derive the low-energy $k \cdot p$ theories of $p_{z}-d_{x^{2}-y^{2}}$ - and $s-p_{z}$-hybridized 2D TIs whose atoms lie at the $1 a$ position of $p 4 / \mathrm{mmm}^{\prime}$, and analytically solve for the bound states on their corners when their edge states are gapped with $p 4 m$-symmetric magnetism. We find that the $p-d-(s-p-)$ hybridized TI evolves into a QI (trivial insulator) when $p 4 m$ symmetric magnetism is introduced, precisely because the inverted bands exhibit different (the same) Kramers pairs of $C_{4 z}$ eigenvalues, such that the symmetry eigenvalues of the occupied bands (do not) match those of a QI in p4m (Supplementary Note 3). We then show in Supplementary Note 9 that the edge states of a $C_{M_{z}}=2$ TCI (such as the $s-d_{x^{2}-y^{2}}$-hybridized TCI phase of Eq. (1)) can gap under an $M_{z}$-breaking, $\mathcal{T}$-symmetric potential (such as Eq. (3)) into four Kramers pairs of corner modes that, if the total system filling is fixed at $1 / 2$, are quarter- (three-quarters-), half-, or fully filled depending on the $C_{4 z}$ eigenvalues of the inverted bulk bands. We then demonstrate that the quarter-filled and three-quartersfilled cases evolve into QIs under $p 4 m$-preserving magnetism, also indicating that the $s$ - $d$-hybridized TCI phase of Eq. (1), like the $p$-d-hybridized 2D TI in Supplementary Notes 5 and 7 , can transition into a QI when its edge states are gapped with $p 4 m$ symmetric, $M_{z}$-breaking magnetism. In Supplementary Note 9 , we explain this by using TQC $22,35,36$ to show that the $s$ - $d$-hybridized TCI phase of Eq. (1) exhibits the same quadrupole moment (modulo $e$ ) as a $p_{z}-d_{x^{2}-y^{2}}$-hybridized 2D TI (when their edge states are gapped by breaking $M_{z}$ and $\mathcal{T}$ ).

3D Dirac semimetals with HOFA states. We now stack the previous $2 \mathrm{D}$ models into $3 \mathrm{D}$ to create physically motivated Hamiltonians modeling solid state materials that are equivalent to tuning cycles of Eqs. (1), (3), and (4) (Fig. 1f, g). In this work, we restrict focus to gapless tuning cycles, which are equivalent $3 \mathrm{D}$ topological semimetals. We begin constructing $3 \mathrm{D}$ models by stacking Eq. (1) in the $z$ direction, adding a term $\left(t_{H} \tau^{z} \cos \left(k_{z}\right)\right)$ that varies the gaps at $k_{x}=k_{y}=0, \pi$ as functions of $k_{z}$, and adding Eq. (4) with a modulation governed by one of two distinct interlayer coupling terms:

$$
\begin{gathered}
\mathcal{H}_{H 1}(\mathbf{k})=\mathcal{H}(\mathbf{k})+U(\mathbf{k})+t_{H} \tau^{z} \cos \left(k_{z}\right), \\
\mathcal{H}_{H 2}(\mathbf{k})=\mathcal{H}(\mathbf{k})+U(\mathbf{k}) \sin \left(k_{z}\right)+t_{H} \tau^{z} \cos \left(k_{z}\right) .
\end{gathered}
$$

In addition to respecting the symmetries of magnetic SG $P 4 \mathrm{~mm}$ (number 99.163 in the BNS notation ${ }^{62}$ ), the space group generated by adding translations in the $z$ direction to ${ }^{62} p 4 m, H_{H 1}(\mathbf{k})$ respects the antiunitary symmetries $M_{z} \times \mathcal{T}$ and $\mathcal{I} \times \mathcal{T}$, whereas $H_{H 2}(\mathbf{k})$ individually respects $M_{z}, \mathcal{I}$, and $\mathcal{T}$ (Table 1 ). To tune $\mathcal{H}_{H 1,2}(\mathbf{k})$ into 3D Dirac semimetal phases, we invert bands by setting $v_{m}<0, t_{1}>0$, and tuning $t_{H}$. When $\left|t_{H}\right|>2 t_{1}+v_{m}$, a pair of Dirac points forms along the $\Gamma Z$ line (Fig. 3c). Viewing $\mathcal{H}\left(k_{x}, k_{y}\right)$ on each constant- $k_{z}$ slice of the $3 \mathrm{D} \mathrm{BZ}$ as a $2 \mathrm{D}$ system, these Dirac points are equivalent to the critical point between trivial and QI phases (Supplementary Note 5). To see this, note that the Dirac points are formed by inverting bands with different pairs of $C_{4 z}$ eigenvalues in a $3 \mathrm{D} \mathrm{BZ}$ for which slices indexed by $k_{z}$ are invariant under magnetic supergroups of $p 4 m$ (Supplementary Note 5). For both Eqs. (5) and (6), the QInontrivial BZ slices are identified by the bulk nested Wilson loop ${ }^{23}$ parameterized as a function of $k_{z}$ (Fig. 3b). When $\left|t_{H}\right|$ is further increased beyond $2 t_{1}-v_{m}$, an additional pair of Dirac points forms along $M A$; we analyze the HOFA-state structure of this semimetal in Supplementary Note 5. We note that similar results were obtained in ref. 53 in toy models of magnetic Dirac semimetals. However, in this work, we also uniquely discover HOFA states in $\mathcal{T}$-symmetric Dirac and Weyl semimetals, allowing their prediction in real materials, which we will address shortly.

We first search for HOFA states in the 3D Dirac semimetal phase of $\mathcal{H}_{H 1}(\mathbf{k})$ (Eq. (5)) that only exhibits a pair of Dirac points along $\Gamma Z$ (specific parameters are listed in Supplementary Note 1). In Eq. (5), $\mathcal{T}$ symmetry is broken, and therefore the 2D Hamiltonians of all $k_{z}$-indexed BZ planes (including $k_{z}=0, \pi$ ) describe either trivial insulators or QIs. Calculating the hinge and surface states of the Dirac semimetal phase of Eq. (5) in a rod geometry that is finite in the $x$ and $y$ directions (Fig. 3f), we observe the absence of $2 \mathrm{D}$ surface states and the presence of HOFAs spanning the projections of the bulk 3D Dirac points along the $1 \mathrm{D}$ hinges. If the bulk Dirac points are gapped by 


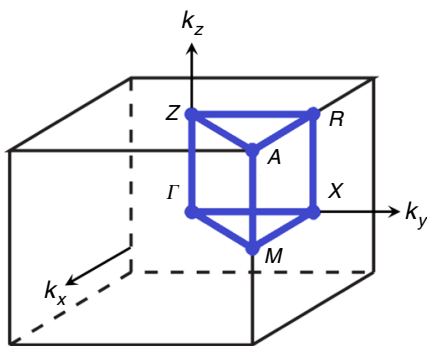

b

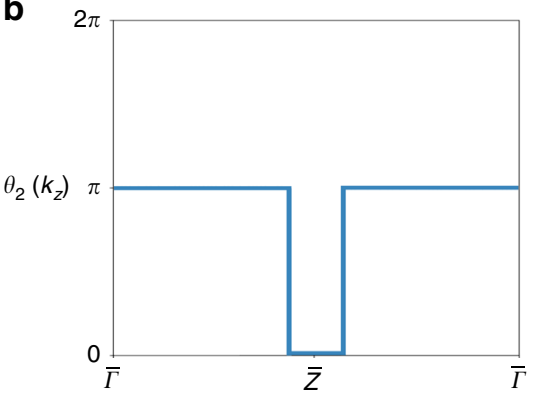

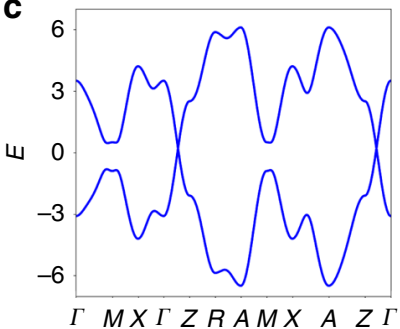

f

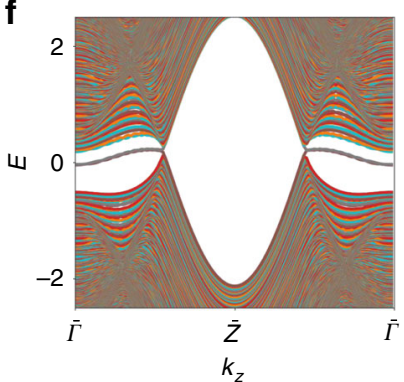

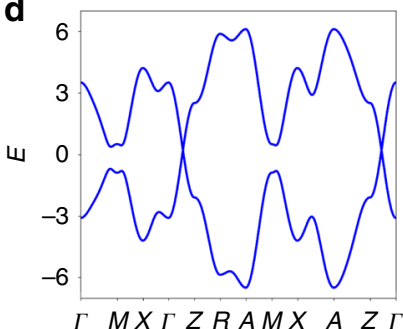

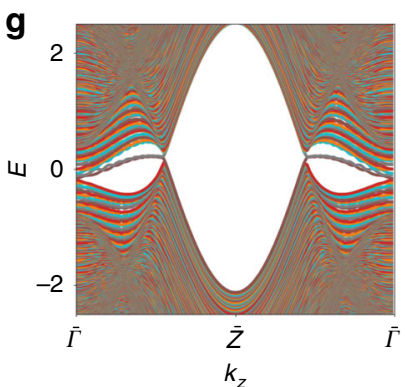

e

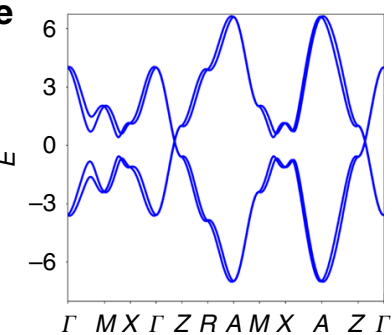

h

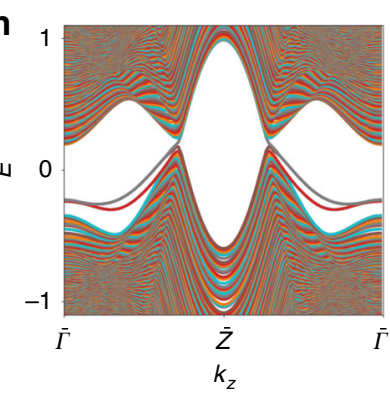

Fig. 3 HOFA states in magnetic, nonmagnetic, and fragile topological Dirac semimetals. a The BZ, $\mathbf{c}$ bulk bands, and $\mathbf{f}$ hinge bands of a $z$-directed rod of a $\mathcal{T}$-broken 3D Dirac semimetal with HOFAs in magnetic SG P4/ $\mathrm{m}^{\prime} \mathrm{mm}$ (Eq. (5)). The Hamiltonian of each $k_{z}$ slice describes either a trivial insulator or a QI, and the bulk Dirac points occur at the quantum critical points between the two phases. $\mathbf{d}$ HOFAs can also be realized in a closely related $\mathcal{T}$-symmetric Dirac semimetal in SG $123 \mathrm{P4} / \mathrm{mmml}^{\prime}$ (Eq. (6)). $\mathbf{g}$ Here, the Hamiltonian of the $k_{z}=0$ plane is in the $2 \mathrm{D} \mathrm{TCl}$ phase ${ }^{10}$ shown in Fig. $2 \mathrm{f}$, $g$, and therefore there is no gap in the hinge-projected surface states at $\bar{\Gamma}$. e Upon breaking $M_{z}$ and $\mathcal{I}$ symmetries by adding Eqs. (3)-(6), which reduces the symmetry to SG 99 $\left(P 4 \mathrm{~mm}^{\prime}\right)$, $\mathbf{h}$ the face $\mathrm{TCl}$ cones gap as they did previously in Fig. 2i-k, resulting in a noncentrosymmetric Dirac semimetal without surface states ${ }^{21}$ whose only topological boundary modes are HOFA states. $\mathbf{h}$ Two sets of weakly split HOFAs meet in Kramers pairs at $\bar{\Gamma}$; if the system filling is fixed to $1 / 2$ (i.e., to the filling of the Dirac points), then one set of HOFA states in $\mathbf{h}$ is half-filled and carries a topological quadrupole moment, and the other set is fully filled, and is topologically trivial. At $\bar{\Gamma}$, this implies that the Kramers pairs of hinge states are three-quarters filled, and that they exhibit the same quadrupole moment (modulo e) as a QI (Supplementary Note 9). The Hamiltonian of the $k_{z}=0$ plane in $\mathbf{e}, \mathbf{h}$ exhibits the same fragile topology as the 2D model in Fig. 2i-k (Supplementary Note 4), implying that the hinge states at $\bar{\Gamma}$ are equivalent to the fractionally charged corner modes of a 2D fragile TI. The model (Eqs. (6) and (3)) shown in $\mathbf{e}, \mathbf{h}$ therefore represents a previously uncharacterized variant of topological semimetal that carries observable signatures of fragile topology. b The 3D semimetallic phases of Eqs. (5) and (6), whether or not Eq. (3) is additionally present, exhibit the same quadrupole-quantized nested Berry phase ${ }^{23} \theta_{2}\left(k_{z}\right)$.

breaking $C_{4 z}$ while preserving mirror symmetry, the HOFA states can evolve into the chiral hinge modes of a $3 \mathrm{D}$ (magnetic) higherorder TI (axion insulator) $24,32,37$. Though $\mathcal{H}_{H 1}(\mathbf{k})$ provides the simplest realization of a HOFA Dirac semimetal without surface states, it also requires the complicated mirror-preserving magnetism of magnetic SG $P 4 / \mathrm{m}^{\prime} \mathrm{mm}$ (123.341 in the BNS notation ${ }^{62}$ ), which cannot be realized in a constant external field or with ferromagnetism. As the number of known magnetic structures is small compared with the number of known materials ${ }^{67}$, it is difficult to identify material candidates for the magnetic HOFA Dirac semimetal phase of $\mathcal{H}_{H 1}(\mathbf{k})$. However, we do find that the antiferromagnetic phase of the Dirac semimetal CeSbTe in magnetic space group $P_{c} 4 / n c c$ (130.432 in the BNS notation ${ }^{62}$ ) is closely related ${ }^{68}$, and may exhibit HOFA states (Supplementary Note 12).
Fortunately, we discover that topological HOFA states are also present in $\mathcal{T}$-symmetric Dirac semimetals. To see this, we tune $\mathcal{H}_{H 2}(\mathbf{k})$ (Eq. (6)) into the parameter regime $2 t_{1}+v_{m}<\left|t_{H}\right|<2 t_{1}-$ $v_{m}$ (specific parameters for Fig. $3 \mathrm{~d}$, g are detailed in Supplementary Note 1) to realize a $\mathcal{T}$-symmetric Dirac semimetal in SG 123 $\left(P 4 / m m m 1^{\prime}\right)$ with a time-reversed pair of Dirac points along $\Gamma Z$ and with mirror Chern number $C_{M_{z}}=2(0)$ at $k_{z}=0(\pi)$. As with the magnetic Dirac semimetal phase of $\mathcal{H}_{H 1}(\mathbf{k})$ (Eq. (5)), the bulk bands of $\mathcal{H}_{H_{2}}(\mathbf{k})$ (Fig. 3d) are twofold degenerate throughout the $\mathrm{BZ}$ as a consequence of $\mathcal{I} \times \mathcal{T}$ symmetry (Table 1 ). Crucially, while this $3 \mathrm{D}$ model $\left(\mathcal{H}_{H_{2}}(\mathbf{k})\right.$ in Eq. (6)) is $\mathcal{T}$-symmetric, 2D planes of the $\mathrm{BZ}$ indexed by $k_{z} \neq 0, \pi$ are still invariant under magnetic layer group $p 4 / \mathrm{m}^{\prime} \mathrm{mm}$, and thus can still be topologically equivalent to QIs. In the Dirac semimetal phase of $\mathcal{H}_{\mathrm{H} 2}(\mathbf{k})$, there are two kinds of topological boundary modes: mirror TCI cones on $M_{z}$-preserving 
$2 \mathrm{D}$ faces at $k_{z}=0$, and singly-degenerate HOFAs on each of the four $1 \mathrm{D}$ hinges connecting the projections of the TCI cones to those of the bulk Dirac points (Fig. 3g). Furthermore, we recognize that $U(\mathbf{k}) \sin \left(k_{z}\right)$ in Eq. (6), which acts in each $k_{z} \neq 0, \pi$ BZ slice like the $p 4 m$-symmetric magnetism (Eq. (4)) depicted in Fig. 2a, is also equivalent to the bulk spin-orbit term previously introduced in ref. ${ }^{21}$ to destabilize the surface Fermi arcs of a Dirac semimetal.

As with the 2D TCI in Fig. 2j, the (100)-surface states of the TCI-nontrivial plane at $k_{z}=0$ of the 3D Dirac semimetal phase of $\mathcal{H}_{H 2}(\mathbf{k})$ can be gapped by breaking $M_{z}$ while preserving $\mathcal{T}$. We accomplish this by adding $V_{M_{z}}(\mathbf{k})$ in Eq. (3) to Eq. (6); this breaks $M_{z}$ and $\mathcal{I}$ while preserving $p 4 m, \mathcal{T}$, and $z$-direction lattice translations, lowering the overall symmetry to SG $99 P 4 m m 1^{\prime}$ (Table 1). In Fig. 3e, h, we respectively plot the bulk and hinge bands of the noncentrosymmetric Dirac semimetal phase resulting from adding Eq. (3) to Eq. (6). We observe that the previous mirror TCI surface states of Eq. (6) have become split and, instead, there are four hinge-localized Kramers pairs of states at $\bar{\Gamma}$ in Fig. 3 h. These eight states become weakly split into two sets of HOFA states at $k_{z} \neq 0$; as described in Supplementary Note 9 , if we fix the overall system filling to $1 / 2$ (i.e., to the filling of the Dirac points), then one of the sets of four HOFA states in Fig. $3 \mathrm{~h}$ is half-filled and carries a topological quadrupole moment, and the other set is fully filled, and is topologically trivial. This implies that the Kramers pairs of hinge states at $\bar{\Gamma}$ in Fig. $3 \mathrm{~h}$ are three-quarters-filled and exhibit the same quadrupole moment (modulo $e$ ) as a QI (Supplementary Note 9). In this noncentrosymmetric Dirac semimetal phase (Eqs. (3) and (6)), the Hamiltonian of the $k_{z}=0$ plane exhibits the same fragile topology as the $2 \mathrm{D}$ insulator in Fig. $2 \mathrm{l}-\mathrm{n}$, and the anomalous, fractionally charged Kramers pairs of states on each hinge at $k_{z}=0$ represent an observable signature of the fragile bands (or of an obstructed atomic limit that can be decomposed into the sum of fragile bands and unobstructed atomic limits ${ }^{37}$ ) (Supplementary Note 9). Therefore, the noncentrosymmetric Dirac semimetal phase of Eqs. (3) and (6) represents a previously uncharacterized fragile topological variant of Dirac semimetal. Like the 3D HOTIs (axion insulators) analyzed in ref. ${ }^{37}$, we refer to this variant of Dirac semimetal (Eqs. (3) and (6)) as fragile because its minimal realization is equivalent to a tuning cycle between a $2 \mathrm{D}$ fragile $\mathrm{TI}$ with anomalous corner modes and a 2D insulator with a trivial Wilson and corner spectrum. Specifically, because the 3D Dirac semimetal phase of Eqs. (3) and (6) respects fourfold rotation and $\mathcal{T}$ symmetries, then the appearance of quarter-empty (or -filled) Kramers pairs of hinge states at $\bar{\Gamma}$ (where the overall system filling is fixed to the filling of the Dirac points) indicates that the occupied bands at $k_{z}=0$ contain the fragile valence bands of the $2 \mathrm{D}$ model described by Eqs. (1) and (3). This occurs because the band inversion that creates the Dirac points along $\Gamma Z$ in Eqs. (3) and (6) also drives the Hamiltonian of the $k_{z}=0$ plane to exhibit the same $C_{4 z}$ eigenvalues as a QI in $p 4 m$ (Supplementary Note 9). While not every fragile phase exhibits intrinsic (anomalous) corner modes (for example, two superposed copies of the $\mathcal{I}$-symmetric fragile TIs examined in refs. ${ }^{32,37}$ combine to form an insulator that is also fragile, but one without anomalous corner states), our results further imply that specific corner states (or state counting imbalances, as discussed in Supplementary Note 9) can still represent a robust signature of a valence manifold that can be decomposed into the sum of unobstructed (trivial) atomic limits and fragile bands, when crystal symmetries and band connectivity are taken into account.

Alternatively, we can formulate a model of a 3D Dirac semimetal from hybridized layers of $p_{z}$ and $d_{x^{2}-y^{2}}$ orbitals in which the Hamiltonian of the $k_{z}=0$ plane instead characterizes a $2 \mathrm{D} \mathrm{TI}^{38}$, as occurs in the experimentally confirmed Dirac semimetals ${ }^{10,18,20,56,57} \mathrm{Cd}_{3} \mathrm{As}_{2}$ and ${ }^{19} \mathrm{Na}_{3} \mathrm{Bi}$. To realize HOFAs as the only boundary (surface and hinge) modes in a semimetal with TI surface cones, unlike with the Dirac semimetal phase of $\mathcal{H}_{H 2}(\mathbf{k})$ (Eq. (6)), one must break $\mathcal{T}$ symmetry (Supplementary Note 7), or apply strain to drive additional band inversions (Supplementary Note 5). In Supplementary Note 5, we also present a model of a $p$ - $d$-hybridized Dirac semimetal with coexisting TI surface states and HOFA hinge states. We also note that the three $\mathcal{T}$-symmetric semimetal models presented in this work-the TCI-nontrivial ${ }^{10}$ Dirac semimetal phase of Eq. (6), the fragile topological Dirac semimetal phase of Eqs. (3) and (6), and the $p-d$-hybridized Dirac semimetal in Supplementary Note 5all exhibit the same number of half-filled HOFA states at $k_{z} \neq 0$ (where the system filling is fixed to the filling of the bulk Dirac points), despite displaying differing numbers of gapped surface states at $k_{z} \neq 0, \pi$. This reinforces the notion that the surface states of Dirac semimetals are not themselves a topological consequence of the bulk Dirac points, but rather only appear due to the topology of high-symmetry planes, and are not required to connect to the surface projections of the bulk Dirac points ${ }^{21}$.

Material realizations. Most surprisingly, the Dirac points in Fig. 3 display the same $k \cdot p$ Hamiltonian as the bulk nodes ${ }^{10,18}$ in the centrosymmetric structural $\left(\alpha\right.$ and $\left.\alpha^{\prime \prime}\right)$ phases of the experimentally established Dirac semimetal ${ }^{18,20,56} \mathrm{Cd}_{3} \mathrm{As}_{2}$ (Fig. 4a). This is because $\mathcal{H}_{\mathrm{H} 2}(\mathbf{k})$ in Eq. (6), which respects symmorphic SG $123 \mathrm{P} 4 / \mathrm{mmml}^{\prime}$, and $\mathrm{Cd}_{3} \mathrm{As}_{2}$ in its room- (high-) temperature $\alpha$ $\left(\alpha^{\prime \prime}\right)$ phase, which respects nonsymmorphic SG $137 \mathrm{P}_{2} / \mathrm{nmcl}^{\prime}$ (SG $142 I 4_{1} /$ acd $1^{\prime}$ ), have little groups along their respective $k_{x}=$ $k_{y}=0$ lines with isomorphic unitary subgroups (Fig. 3a and Supplementary Notes 5 and 12). Both the $\alpha$ and $\alpha^{\prime \prime}$ structural phases of $\mathrm{Cd}_{3} \mathrm{As}_{2}$ exhibit the same bulk topology-they both host a time-reversed pair of Dirac points along $k_{x}=k_{y}=0$, and are equivalent at $k_{z}=0$ to $2 \mathrm{D}$ TIs due to a band inversion between the $5 s$ orbitals of $\mathrm{Cd}$ and the $m_{j}= \pm 3 / 2$ subset of the $4 p_{x, y}$ orbitals of $A s^{18}$. In terms of the $s-p$ - and $s$ - $d$-hybridized semimetals and TIs analyzed in Supplementary Notes 5-9, the topology of the $\alpha$ and $\alpha^{\prime \prime}$ structural phases of $\mathrm{Cd}_{3} \mathrm{As}_{2}$ can be understood by noting that the $m_{j}= \pm 3 / 2$ subset of spinful $p_{x, y}$ orbitals exhibits the same parity eigenvalues as spinful $p_{z}$ orbitals and the same fourfold rotation eigenvalues as spinful $d_{x^{2}-y^{2}}$ orbitals ${ }^{22}$. This implies that the bulk topology of $\mathrm{Cd}_{3} \mathrm{As}_{2}$ (Supplementary Note 13) is equivalent to the superposition of an $s-p_{z^{-}}$ hybridized 3D TI and an $s-d_{x^{2}-y^{2}}$-hybridized topological Dirac semimetal with HOFA states (or equivalently, to the $p_{z}-d_{x^{2}-y^{2}}$ HOFA Dirac semimetal in Supplementary Note 5). Using an analytic formulation of topological (intrinsic) HOFA states derived in Supplementary Notes 6-10, we find that the $k \cdot p$ theory and symmetries of $\alpha-\mathrm{Cd}_{3} \mathrm{As}_{2}$ imply the presence of HOFA states on the hinges of (001)- $(z-)$ axis-directed samples, which have recently been synthesized in experiment ${ }^{69}$. Though the $\alpha$ phase is body-centered and respects $x$ - and $y$-normal glide reflections, instead of $M_{x, y}$ like Eq. (6), we provide proofs in Supplementary Note 12 demonstrating that body-centered and glide-symmetric Dirac semimetals also exhibit topological HOFA states like those in Fig. $3 \mathrm{f}-\mathrm{h}$.

The symmetries and $k \cdot p$ theory of the Dirac points of Eq. (6) additionally imply that the primitive tetragonal $\left(\alpha^{\prime \prime}\right)$ phase of $\mathrm{Cd}_{3} \mathrm{As}_{2}$ should also exhibit topological HOFA states. Although the $\alpha^{\prime \prime}$ phase naturally occurs at high temperatures $\left(475-600{ }^{\circ} \mathrm{C}\right)^{56}$, it can be stabilized in single crystalline form at room temperature and below by $2 \%$ zinc doping 57 ; as $\mathrm{Zn}$ is isoelectronic to $\mathrm{Cd}$, this doping will not affect the Fermi level. Calculating the hinge spectrum of the original $k \cdot p$ model introduced in ref. ${ }^{18}$ for $\alpha$ "- $-\mathrm{Cd}_{3} \mathrm{As}_{2}$, we confirm our prediction of previously overlooked 
a
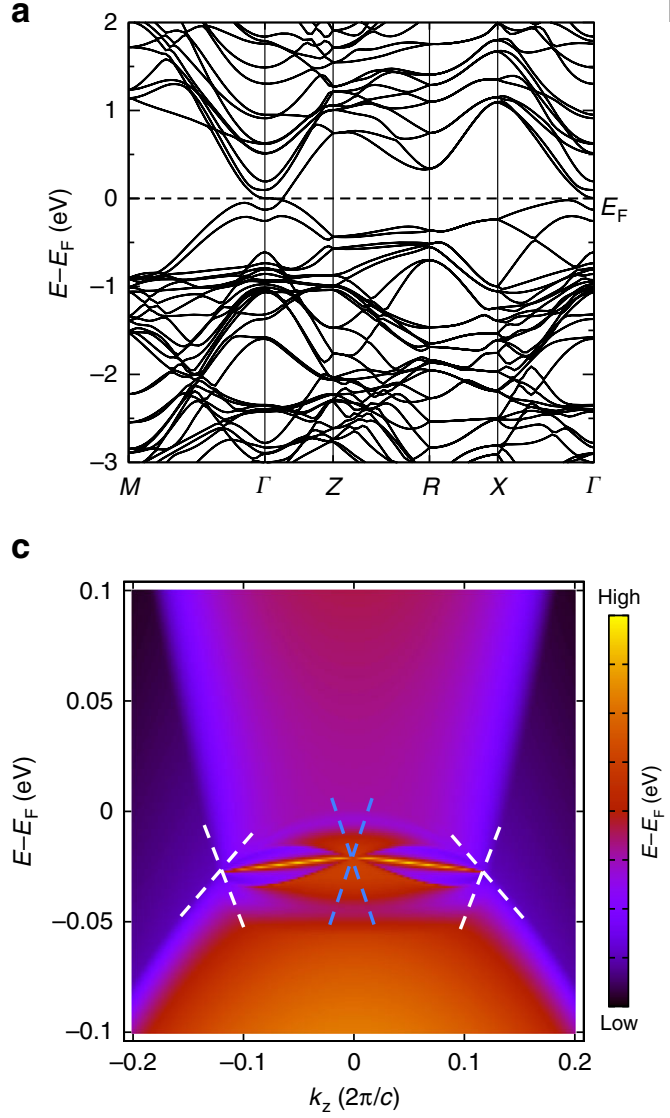

b
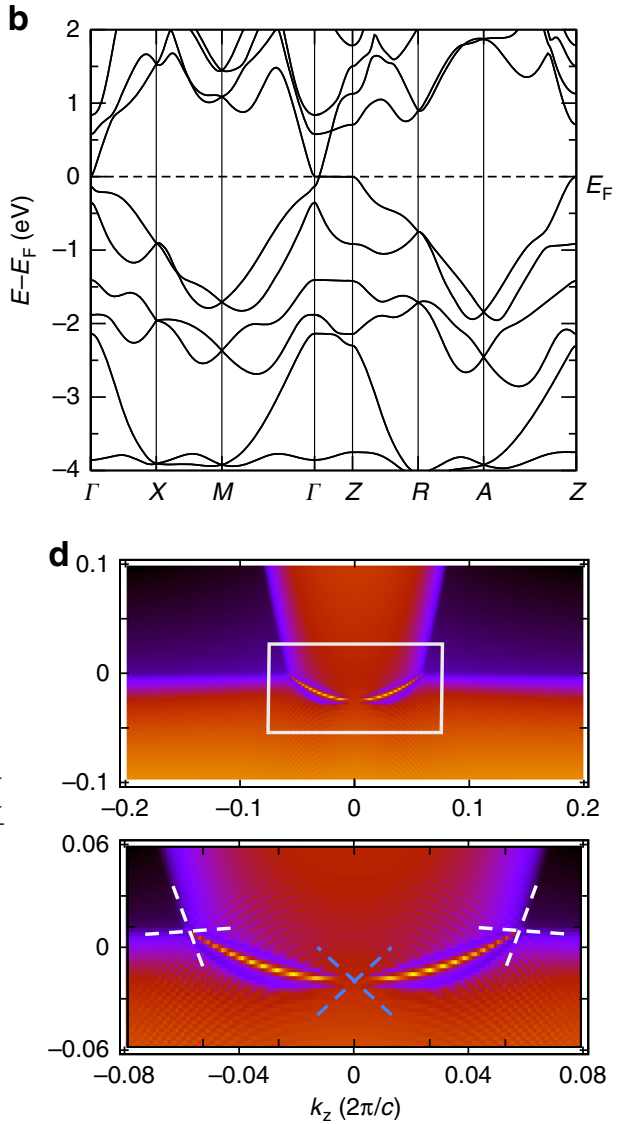

Fig. 4 HOFA states in $\boldsymbol{\alpha}^{\prime \prime}-\mathbf{C d}_{\mathbf{3}} \mathbf{A} \mathbf{s}_{\mathbf{2}}$ and $\mathbf{K M g B i}$. a Bulk bands incorporating the effects of SOC of $\alpha^{\prime \prime}-\mathrm{Cd}_{3} \mathrm{As}_{2}$ in SG 137 $\left(P 4_{2} / n m c 1^{\prime}\right)^{10,18,20,56,57}$. This semimetal exhibits $\mathcal{T}$-reversed pairs of bulk $3 \mathrm{D}$ Dirac cones as well as $2 \mathrm{D}$ TI cones on its faces as a consequence of the nontrivial $\mathbb{Z}_{2}$ topology of the $k_{z}=0$ plane (Supplementary Note 13) ${ }^{10,18-20}$. c The hinge spectrum of the $k \cdot p$ model of $\alpha^{\prime \prime}-\mathrm{Cd}_{3} \mathrm{As}_{2}$ introduced in ref. ${ }^{18}$ exhibits previously undetected HOFA states connecting the projections of the bulk Dirac cones (white) to the hinge projection of the topological face cones (blue). b Bulk bands incorporating the effects of SOC and (d) hinge states of the candidate tilted Dirac semimetal KMgBi in SG $129\left(P 4 / n m m 1^{\prime}\right)^{58,59}$. $\mathbf{d}$ Zooming into the green boxed region, HOFAs are clearly visible connecting the projections of the bulk, tilted 3D Dirac points (white) to the projections of surface 2D TI cones (blue). The bulk band structures in (a) and (b) were obtained from first-principles, and then used to fit tight-binding models whose hinge Green's functions are shown in (c) and (d), respectively (Supplementary Note 13).

HOFAs (Fig. 4c and Supplementary Note 13). This suggests a clear route towards predicting additional candidate Dirac semimetals with HOFA states: using the low-energy theory of the QI (Supplementary Note 6), we determine that strong-SOC Dirac semimetals with SGs that contain point group $4 \mathrm{~mm}\left(C_{4 v}\right)$ will exhibit HOFA states when they are cut into nanorods or exhibit step edge configurations that preserve fourfold axes (Table 2). This is analogous to the helical hinge modes in the HOTI bismuth, which are only observable in samples that are cut into nanowires (or terminated with step edge configurations) that preserve bulk rotation and $\mathcal{I}$ symmetries ${ }^{31}$. A number of candidate Dirac semimetals have already been identified in the SGs in Table 2, including the aforementioned $\alpha$ and $\alpha^{\prime \prime}$ phases of $\mathrm{Cd}_{3} \mathrm{As}_{2}$, the rutile-structure $\left(\beta^{\prime}-\right)$ phase of $\mathrm{PtO}_{2}$ in SG $136\left(P 4_{2} / m n m 1^{\prime}\right)^{60,61}$, and families of tilted Dirac semimetals related to $\mathrm{VAl}_{3}$ in SG 139 $\left(I 4 / m m m 1^{\prime}\right)^{70}, \mathrm{YPd}_{2} \mathrm{Sn}$ in SG $225\left(F m \overline{3} m 1^{\prime}\right)^{71}$, and KMgBi in SG $129\left(P 4 / n m m 1^{\prime}\right)^{58,59}$.

Of the candidate HOFA semimetals that we identified, we highlight $\mathrm{KMgBi}$ and $\beta^{\prime}-\mathrm{PtO}_{2}$ because of their simple geometries. $\mathrm{KMgBi}$ has recently been identified as a topological semimetal with critically tilted bulk Dirac $\operatorname{cones}^{58}$, and its electronic properties have been examined in experiment ${ }^{59}$. In Fig. 4, we plot the bulk bands (b) calculated from first principles, and the hinge spectrum (d) of a lattice tight-binding model of $\mathrm{KMgBi}$ fit to
Table 2 Space groups (SGs) that admit Dirac points with HOFA states derived from the QI introduced in ref. ${ }^{23}$.

\section{Space groups admitting dirac points with HOFA states}

\begin{tabular}{lll}
\hline Point group name & Point group symbol & SG numbers \\
\hline$C_{4 v}$ & $4 m m 1^{\prime}$ & $99-110$ \\
$D_{4 h}$ & $4 / m m m 1^{\prime}$ & $123-142$ \\
$O_{h}$ & $m \overline{3} m 1^{\prime}$ & $221-230$
\end{tabular}

All of these SGs have point groups that contain C4v. We obtain this list of SGs by combining the nested Jackiw-Rebbi formulation of the QI in Supplementary Note 6 with an analysis of the crystallographic rod groups in Supplementary Note 12. In all of these SGs, semimetals with Dirac points along lines with $4 \mathrm{~mm}$ or $4 / \mathrm{m}^{\prime} \mathrm{mm}$ symmetry will exhibit intrinsic HOFA states when cut into nanorods that preserve fourfold axes and are thick compared with the in-plane lattice spacing. This list is a complete enumeration of the SGs that permit Dirac semimetals with HOFA states directly related to the QI introduced in ref. ${ }^{23}$; alternative realizations of Dirac and Weyl semimetals with HOFA states derived from other 2D magnetic insulators with corner states are also possible (refs. ${ }^{32,37}$ and Supplementary Note 12).

the bands in (b) (Supplementary Note 13). We find that the $k_{z}=0$ plane of KMgBi exhibits the topology of a 2D TI (Supplementary Note 13), in agreement with the surface-state calculation in ref. ${ }^{58}$. In the vicinity of $k_{z}=0$ (Fig. 4c), HOFAs are clearly visible connecting the hinge projections of the bulk 3D Dirac points (white) to the projections of $2 \mathrm{D}$ surface TI cones at $k_{z}=0$ (blue). 

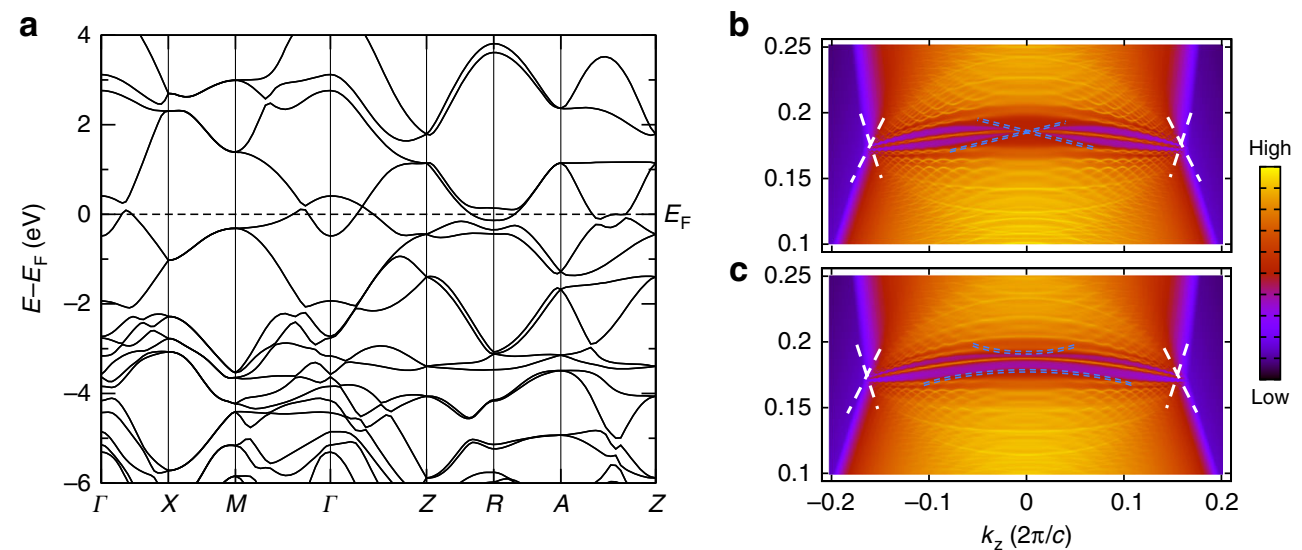

Fig. 5 HOFA states and fragile Dirac semimetal phase in $\boldsymbol{\beta}^{\prime}-\mathbf{P t O}_{\mathbf{2}}$. a Bulk bands incorporating the effects of SOC of the candidate Dirac semimetal $\beta^{\prime}-\mathrm{PtO}{ }_{2}$ in SG $136\left(\mathrm{PL}_{2} / \mathrm{mnml}^{\prime}\right)^{60,61}$. Unlike in the Dirac semimetals $\alpha^{\prime \prime}-\mathrm{Cd}_{3} \mathrm{As}_{2}$ and $\mathrm{KMgBi}$ examined in Fig. 4, the $k_{z}=0$ plane of $\beta^{\prime}-\mathrm{PtO} \mathrm{O}_{2}$ is equivalent to a $2 \mathrm{D}$ TCl with mirror Chern number $C_{M_{2}}=2$ (ref. ${ }^{61}$ and Supplementary Note 13). b The hinge spectrum of $\beta^{\prime}-\mathrm{PtO}_{2}$ exhibits two narrowly split $\mathrm{HOFA}$ states connecting the hinge projections of the bulk 3D Dirac points (white) to the projections of two surface TCl cones at $k_{z}=0$ (blue). Fixing the system filling to that of the bulk Dirac points, we find that the lower HOFA state in energy in (b) is half-filled, and therefore exhibits a topological quadrupole moment, and the higher state is unoccupied, and is thus topologically trivial, as discussed in Supplementary Notes 9 and 13. c In the presence of a $z$-directed external electric field, the surface $\mathrm{TCl}$ cones in $\beta^{\prime}-\mathrm{PtO}_{2}$ become gapped (blue), allowing for the two HOFA states to meet at $k_{z}=0$ in a quarter-filled Kramers pair of corner modes that is characteristic of the fragile phase introduced in this work (Figs. 2i-k, 3e, h, and Supplementary Notes 4, 9, and 13). The bulk band structure in (a) was obtained from first-principles, and then used to fit a tight-binding model whose hinge Green's functions in the absence and presence of an external electric field are shown in (b) and (c), respectively (Supplementary Note 13).

This boundary mode structure is captured by the model of a $p-d$ hybridized Dirac semimetal in Supplementary Note 5.

In Fig. 5, we also examine the bulk and hinge spectra of the candidate Dirac semimetal $\beta^{\prime}-\mathrm{PtO}_{2}$. Single crystals of $\mathrm{PtO}_{2}$ in its rutile-structure $\left(\beta^{\prime}\right)$ phase have previously been prepared in experiment ${ }^{60}$, and its bulk and surface electronic structure were examined in a previous theoretical work ${ }^{61}$. In $\beta^{\prime}-\mathrm{PtO}_{2}$, the Hamiltonian of the $k_{z}=0$ plane is equivalent to a $2 \mathrm{D}$ TCI with mirror Chern number $C_{M_{z}}=2$; therefore $\beta^{\prime}-\mathrm{PtO}_{2}$ is more closely related to the $s$ - $d$-hybridized HOFA semimetal model introduced in this work (Eq. (6)) than it is to $\alpha^{\prime \prime}-\mathrm{Cd}_{3} \mathrm{As}_{2}$ and $\mathrm{KMgBi}$, which at $k_{z}=0$ are instead equivalent to $2 \mathrm{D}$ TIs (Fig. 4 and Supplementary Note 13). In Fig. 5, we plot the bulk bands (a) and hinge spectrum (b) of $\beta^{\prime}-\mathrm{PtO}_{2}$ calculated from first principles, as detailed in Supplementary Note 13. In the spectrum of a single hinge (Fig. 5b), we observe two narrowly split HOFA states connecting the hinge projections of the bulk 3D Dirac points to the projections of the surface 2D TCI cones. Fixing the system filling to that of the bulk Dirac points, we observe that, similar to the hinge spectrum of the fragile topological Dirac semimetal in Fig. $3 \mathrm{~h}$, only one of the HOFA states on each hinge of $\beta^{\prime}-\mathrm{PtO}_{2}$ is half-filled. Specifically, we find that the lower HOFA state in energy in Fig. 5b is half filled, and therefore carries a topological quadrupole moment (Supplementary Note 9), and that the other HOFA state is unoccupied, and is therefore topologically trivial.

Because the TCI surface cones in $\beta^{\prime}-\mathrm{PtO}_{2}$ are only protected by $M_{z}$ symmetry, they can be gapped without breaking $\mathcal{T}$ symmetry, unlike the 2D TI surface cones in $\alpha^{\prime \prime}-\mathrm{Cd}_{3} \mathrm{As}_{2}$ and $\mathrm{KMgBi}$ (Supplementary Note 7). To preserve the bulk Dirac points and intrinsic HOFA states in $\beta^{\prime}-\mathrm{PtO}_{2}$ while gapping the surface TCI cones, we must break $M_{z}$ (and hence $\mathcal{I}$ ) symmetry while preserving the $4_{2}$ screw, $x$-directed $n$-glide reflection, $\mathcal{T}$, and lattice translation symmetries of SG $136\left(P 4_{2} / m n m 1^{\prime}\right)$. Though this cannot be accomplished by uniaxial strain, which either manifests as symmetry-preserving stretching in the $z$ direction or as a translation-breaking strain gradient, these symmetry requirements can be satisfied in experiment by applying an external electric field that is spatially constant (or slowly varying on the scale of the lattice spacing) along the $z-(c-)$ axis of a fourfold-symmetric $\beta^{\prime}-\mathrm{PtO}_{2}$ sample. Implementing the effects of an external electric field into our Green's function calculation of the hinge states in $\beta^{\prime}-\mathrm{PtO}_{2}$ (Fig. 5c), we observe that the TCI cones have become gapped, and that the HOFA states instead meet in a Kramers pair of quarter-filled corner modes at $k_{z}=0$.

Furthermore, because the $k_{z}=0$ plane of $\beta^{\prime}-\mathrm{PtO}_{2}$ both exhibits the topology of a $C_{M_{z}}=2 \mathrm{TCI}$ and carries the same bulk fourfold rotation eigenvalues as a QI in $p 4 m$ (Supplementary Note 3), then the quarter-filled corner modes that appear at $k_{z}=0$ in the hinge spectrum of $\beta^{\prime}-\mathrm{PtO}_{2}$ when its TCI surface states are gapped with an external electric field (Fig. 5c) indicate that the valence manifold at $k_{z}=0$ can be separated into trivial bands and fragile bands with the same topology as the $2 \mathrm{D}$ fragile phase introduced in this work (Eqs. (1) and (3)). Specifically, $\beta^{\prime}-\mathrm{PtO}_{2}$ only differs from a trivial (unobstructed) atomic limit without Dirac points or hinge states by a single inversion at the $\Gamma$ point between bands with the same parity eigenvalues and different fourfold rotation eigenvalues (Fig. 5a and Supplementary Note 13). Therefore, the band inversion in $\beta^{\prime}-\mathrm{PtO}_{2}$ drives the $k_{z}=0$ plane into the same $C_{M_{z}}=2$ TCI phase as that of Eq. (1), which necessarily gaps into an insulator with fragile bands and fractionally charged Kramers pairs of corner modes when $M_{z}$ is relaxed while preserving fourfold rotation and $\mathcal{T}$ (Supplementary Note 9). Whether the entire valence manifold at $k_{z}=0$ is fragile or an obstructed atomic limit depends on the precise details of the bands below the Fermi energy, and for the case of the fragile phase introduced in this work (Eqs. (1) and (3)), uniquely cannot be inferred from the symmetry eigenvalues of the occupied bands (Supplementary Note 4), unlike the fragile phases examined in previous works $^{33-37}$. Nevertheless, like the $\mathcal{I}$-symmetric fragile phases with corner modes introduced in refs. ${ }^{32,37}$, the fragile phase of Eqs. (1) and (3) still exhibits anomalous (intrinsic) corner modes when trivial bands (i.e., unobstructed atomic limits without corner states) are introduced below the Fermi energy. Therefore, because the $k_{z}=0$ plane of $\beta^{\prime}-\mathrm{PtO}_{2}$ can be decomposed into a set of trivial bands without corner states and the inverted bands at the Fermi 
energy, it still exhibits the fractionally charged corner states shown in Fig. $5 c$ when $M_{z}$ is relaxed while preserving $\mathcal{T}$ and fourfold rotation, whether or not the entire valence manifold at $k_{z}=0$ is fragile or an obstructed atomic limit. We draw further connection between $\beta^{\prime}-\mathrm{PtO}_{2}$ and the model of an $s$ - $d$-hybridized, noncentrosymmetric, fragile topological Dirac semimetal introduced in this work (Eqs. (3) and (6) and Fig. 3e, h) by noting that the quarter-filled corner modes at $k_{z}=0$ in Fig. $5 \mathrm{c}$ represent the particle-hole conjugates of the three-quarters-filled fragile-phase corner modes observable at $k_{z}=0$ in Fig. 3h (Supplementary Notes 9 and 13).

\section{Discussion}

The HOFA states introduced in this work may be detectable through transport and STM experiments ${ }^{31}$. Though our analysis has focused on nanowire geometries, HOFA states may also be observable through momentum-resolved probes of fourfoldsymmetric arrangements of step edges on the surfaces of Dirac semimetals with the SGs in Table 2. Nonlocal quantum oscillation experiments ${ }^{72}$ and SQUID measurements ${ }^{31}$ performed on materials with HOFA states are likely to show interesting signatures reflecting the reduced dimensionality of the hinge modes. By generalizing the analysis performed in this work, further examples of topological semimetals with HOFA states should be readily discoverable, including HOFA Dirac semimetals with sixfold symmetries and, as discussed in Supplementary Notes 10 and 12, high-fold-rotation Weyl semimetals with coexisting surface Fermi arcs and HOFA states. In addition, our atomic-orbital description of QIs with $s-d$ hybridization suggests the possibility of quadrupolar generalizations of polyacetylene ${ }^{16,43}$. Finally, because the analytic expression that we obtain for the bound (corner) states of the QI in Supplementary Note 7, when the reflection symmetries of $p 4 m$ are relaxed, can be expressed as the superposition of $1+$ $2 n$ (i.e., an odd number) of quadrupole moments whose direction is a free parameter but whose magnitude is fixed to $e / 2$ (Supplementary Note 10), then it bears similarities with recent gaugetheory descriptions of fractons with anomalous tensor charges ${ }^{73}$.

\section{Methods}

All tight-binding, surface state, hinge state, and Wilson loop calculations were performed using the standard implementation of the open-source PYTHTB Python package ${ }^{74}$. Nested Wilson loop calculations were performed using an extension of PYTHTB that is documented in ref. ${ }^{37}$.

First-principles calculations were performed using the projector augmented wave $^{75}$ method as implemented in the Vienna $\mathrm{Ab}$ initio Simulation Package ${ }^{76,77}$. The hinge states of $\alpha^{\prime \prime}-\mathrm{Cd}_{3} \mathrm{As}_{2}, \mathrm{KMgBi}$, and $\beta^{\prime}-\mathrm{PtO}_{2}$ were obtained by mapping the bands closest to the Fermi energy to tight-binding models and then calculating the Green's function along a single 1D hinge of a slab that was infinite along the crystal axis parallel to the hinge and respectively finite and semi-infinite along the two perpendicular axes. Further details of our first-principles and hinge Green's function calculations are provided in Supplementary Note 13.

\section{Data availability}

The data supporting the findings of this study are available within the paper and other findings of this study are available from the corresponding authors upon reasonable request. All first-principles calculations were performed using CIF structure files with the experimental lattice parameters, which can be obtained from the Inorganic Crystal Structure Database (ICSD) ${ }^{78}$ using the accession numbers provided in Supplementary Note 13.

Received: 23 October 2019; Accepted: 15 December 2019; Published online: 31 January 2020

\section{References}

1. Castro Neto, A. H., Guinea, F., Peres, N. M. R., Novoselov, K. S. \& Geim, A. K. The electronic properties of graphene. Rev. Mod. Phys. 81, 109-162 (2009).
2. Wan, X., Turner, A. M., Vishwanath, A. \& Savrasov, S. Y. Topological semimetal and fermi-arc surface states in the electronic structure of pyrochlore iridates. Phys. Rev. B 83, 205101 (2011).

3. Weng, H., Fang, C., Fang, Z., Bernevig, B. A. \& Dai, X. Weyl semimetal phase in noncentrosymmetric transition-metal monophosphides. Phys. Rev. X 5, 011029 (2015).

4. $\mathrm{Xu}, \mathrm{S}$.-Y. et al. Discovery of a weyl fermion semimetal and topological fermi arcs. Science 349, 613-617 (2015).

5. Wieder, B. J., Kim, Y., Rappe, A. M. \& Kane, C. L. Double dirac semimetals in three dimensions. Phys. Rev. Lett. 116, 186402 (2016).

6. Bradlyn, B. et al. Beyond dirac and weyl fermions: unconventional quasiparticles in conventional crystals. Science 353, aaf5037 (2016).

7. Chang, G. et al. Unconventional chiral fermions and large topological fermi arcs in RhSi. Phys. Rev. Lett. 119, 206401 (2017).

8. Tang, P., Zhou, Q. \& Zhang, S.-C. Multiple types of topological fermions in transition metal silicides. Phys. Rev. Lett. 119, 206402 (2017).

9. Chang, G. et al. Topological quantum properties of chiral crystals. Nat. Mater 17, 978-985 (2018).

10. Yang, B.-J. \& Nagaosa, N. Classification of stable three-dimensional dirac semimetals with nontrivial topology. Nat. Comm. 5, 4898 (2014).

11. Kim, Y., Wieder, B. J., Kane, C. L. \& Rappe, A. M. Dirac line nodes in inversion-symmetric crystals. Phys. Rev. Lett. 115, 036806 (2015).

12. Schröter, N. B. M. et al. Chiral topological semimetal with multifold band crossings and long fermi arcs. Nat. Phys. 15, 759-765 (2019).

13. Takane, D. et al. Observation of chiral fermions with a large topological charge and associated fermi-arc surface states in CoSi. Phys. Rev. Lett. 122, 076402 (2019).

14. Sanchez, D. S. et al. Topological chiral crystals with helicoid-arc quantum states. Nature 567, 500-505 (2019).

15. Rao, Z. et al. Observation of unconventional chiral fermions with long fermi arcs in CoSi. Nature 567, 496-499 (2019).

16. Su, W. P., Schrieffer, J. R. \& Heeger, A. J. Solitons in polyacetylene. Phys. Rev. Lett. 42, 1698-1701 (1979).

17. Rice, M. J. \& Mele, E. J. Elementary excitations of a linearly conjugated diatomic polymer. Phys. Rev. Lett. 49, 1455-1459 (1982).

18. Wang, Z., Weng, H., Wu, Q., Dai, X. \& Fang, Z. Three-dimensional dirac semimetal and quantum transport in $\mathrm{Cd}_{3} \mathrm{As}_{2}$. Phys. Rev. B 88, 125427 (2013).

19. $\mathrm{Xu}, \mathrm{S}$.-Y. et al. Observation of fermi arc surface states in a topological metal. Science 347, 294-298 (2015).

20. $\mathrm{Yi}, \mathrm{H}$. et al. Evidence of topological surface state in three-dimensional dirac semimetal $\mathrm{Cd}_{3} \mathrm{As}_{2}$. Sci. Rep. 4, $6106 \mathrm{EP}-(2014)$.

21. Kargarian, M., Randeria, M. \& Lu, Y.-M. Are the surface fermi arcs in dirac semimetals topologically protected? Proc. Natl Acad. Sci. 113, 8648-8652 (2016).

22. Bradlyn, B. et al. Topological quantum chemistry. Nature 547, 298 EP (2017).

23. Benalcazar, W. A., Bernevig, B. A. \& Hughes, T. L. Quantized electric multipole insulators. Science 357, 61-66 (2017).

24. Benalcazar, W. A., Bernevig, B. A. \& Hughes, T. L. Electric multipole moments, topological multipole moment pumping, and chiral hinge states in crystalline insulators. Phys. Rev. B 96, 245115 (2017).

25. Schindler, F. et al. Higher-order topological insulators. Sci Adv. 4, eaat0346 (2018).

26. Song, Z., Fang, Z. \& Fang, C. (d-2)-dimensional edge states of rotation symmetry protected topological states. Phys. Rev. Lett. 119, 246402 (2017)

27. Langbehn, J., Peng, Y., Trifunovic, L., vonOppen, F. \& Brouwer, P. W. Reflection-symmetric second-order topological insulators and superconductors. Phys. Rev. Lett. 119, 246401 (2017).

28. Wieder, B. J. et al. Wallpaper fermions and the nonsymmorphic dirac insulator. Science 361, 246-251 (2018)

29. Fang, C. \& Fu, L. New classes of topological crystalline insulators having surface rotation anomaly. Science Advances 5, eaat2374 (2019).

30. Khalaf, E., Po, H. C., Vishwanath, A. \& Watanabe, H. Symmetry indicators and anomalous surface states of topological crystalline insulators. Phys. Rev. X 8, 031070 (2018).

31. Schindler, F. et al. Higher-order topology in bismuth. Nat. Phys. 14, 918-924 (2018).

32. Wang, Z., Wieder, B. J., Li, J., Yan, B. \& Bernevig, B. A. Higher-order topology, monopole nodal lines, and the origin of large fermi arcs in transition metaldichalcogenides $X \mathrm{Te}_{2}(x=\mathrm{Mo}, \mathrm{W})$. Phys. Rev. Lett. 123, 186401 (2019).

33. Po, H. C., Watanabe, H. \& Vishwanath, A. Fragile topology and wannier obstructions. Phys. Rev. Lett. 121, 126402 (2018).

34. Alexandradinata, A., Dai, X. \& Bernevig, B. A. Wilson-loop characterization of inversion-symmetric topological insulators. Phys. Rev. B. 89, 155114 (2014).

35. Cano, J. et al. Topology of disconnected elementary band representations. Phys. Rev. Lett. 120, 266401 (2018).

36. Bradlyn, B., Wang, Z., Cano, J. \& Bernevig, B. A. Disconnected elementary band representations, fragile topology, and wilson loops as topological indices: An example on the triangular lattice. Phys. Rev. B 99, 045140 (2019). 
37. Wieder, B. J. \& Bernevig, B. A. The axion insulator as a pump of fragile topology. Preprint at https://arxiv.org/abs/1810.02373 (2018).

38. Bernevig, B. A., Hughes, T. L. \& Zhang, S.-C. Quantum spin hall effect and topological phase transition in HgTe quantum wells. Science 314, 1757-1761 (2006).

39. Kane, C. L. \& Mele, E. J. Quantum spin hall effect in graphene. Phys. Rev. Lett. 95, 226801 (2005)

40. Thouless, D. J. Wannier functions for magnetic sub-bands. J Phys C: Solid State Phys. 17, L325 (1984).

41. Soluyanov, A. A. \& Vanderbilt, D. Smooth gauge for topological insulators. Phys. Rev. B 85, 115415 (2012).

42. King-Smith, R. D. \& Vanderbilt, D. Theory of polarization of crystalline solids. Phys. Rev. B 47, 1651-1654 (1993).

43. Chiang, C. K. et al. Electrical conductivity in doped polyacetylene. Phys. Rev. Lett. 39, 1098-1101 (1977).

44. Ruffieux, P. et al. On-surface synthesis of graphene nanoribbons with zigzag edge topology. Nature 531, 489 EP (2016).

45. Topp, A. et al. Surface floating $2 \mathrm{~d}$ bands in layered nonsymmorphic semimetals: ZrSiS and related compounds. Phys. Rev. X 7, 041073 (2017).

46. Takane, D. et al. Observation of dirac-like energy band and ring-torus fermi surface associated with the nodal line in topological insulator CaAgAs. NPJ Quantum Mater. 3, 1 (2018).

47. Fu, L. \& Kane, C. L. Topological insulators with inversion symmetry. Phys. Rev. B 76, 045302 (2007).

48. Po, H. C., Vishwanath, A. \& Watanabe, H. Symmetry-based indicators of band topology in the 230 space groups. Nat. Commun. 8, 50 (2017).

49. Song, Z., Zhang, T., Fang, Z. \& Fang, C. Quantitative mappings between symmetry and topology in solids. Nat. Commun. 9, 3530 (2018).

50. Kruthoff, J., de Boer, J., van Wezel, J., Kane, C. L. \& Slager, R.-J. Topological classification of crystalline insulators through band structure combinatorics. Phys. Rev. X 7, 041069 (2017).

51. Serra-Garcia, M. et al. Observation of a phononic quadrupole topological insulator. Nature 555, 342-345 (2018).

52. Jackiw, R. \& Rebbi, C. Solitons with fermion number 1/2. Phys. Rev. D 13, 3398-3409 (1976).

53. Lin, M. \& Hughes, T. L. Topological quadrupolar semimetals. Phys. Rev. B 98 , 241103 (2018).

54. Călugăru, D., Juričić, V. \& Roy, B. Higher-order topological phases: a general principle of construction. Phys. Rev. B 99, 041301 (2019).

55. Kitaev, A. Periodic table for topological insulators and superconductors. AIP Conf. Proc. 1134, 22-30 (2009).

56. Ali, M. N. et al. The crystal and electronic structures of $\mathrm{Cd}_{3} \mathrm{As}_{2}$, the threedimensional electronic analogue of graphene. Inorganic Chem. 53, 4062-4067 (2014).

57. Arushanov, E. Crystal growth and characterization of $\mathrm{ii}_{3} \mathrm{v}_{2}$ compounds. Prog. Cryst. Growth Charact. 3, 211-255 (1980).

58. Le, C. et al. Three-dimensional topological critical diracsemimetal in $a \mathrm{MgBi}$ $(a=\mathrm{K}, \mathrm{Rb}, \mathrm{Cs})$. Phys. Rev. B 96, 115121 (2017).

59. Zhang, X., Sun, S. \& Lei, H. Narrow-gap semiconducting properties of KMgBi with multiband feature. Phys. Rev. B 95, 035209 (2017).

60. Schwartz, K., Gillson, J. \& Shannon, R. Crystal growth of $\mathrm{CdPt}_{3} \mathrm{O}_{6}, \mathrm{MnPt}_{3} \mathrm{O}_{6}$, $\mathrm{CoPt}_{3} \mathrm{O}_{6}$ and $\mathrm{B}-\mathrm{PtO}_{2}$. J. Cryst. Growth 60, 251-254 (1982).

61. Kim, R., Yang, B.-J. \& Kim, C. H. Crystalline topological dirac semimetal phase in rutile structure $\beta^{\prime}-\mathrm{PtO}_{2}$. Phys. Rev. B 99, 045130 (2019).

62. Litvin, D. B. Magnetic Group Tables (International Union of Crystallography, 2013).

63. Fidkowski, L., Jackson, T. S. \& Klich, I. Model characterization of gapless edge modes of topological insulators using intermediate brillouin-zone functions. Phys. Rev. Lett. 107, 036601 (2011).

64. Fu, L. Topological crystalline insulators. Phys. Rev. Lett. 106, 106802 (2011).

65. Teo, J. C. Y., Fu, L. \& Kane, C. L. Surface states and topological invariants in three-dimensional topological insulators: Application to $B i_{1-x} S b_{x}$. Phys. Rev. $B$ 78, 045426 (2008).

66. Liu, J., Qian, X. \& Fu, L. Crystal field effect induced topological crystalline insulators in monolayer iv-vi semiconductors. Nano Lett. 15, 2657-2661 (2015).

67. Gallego, S. V. et al. MAGNDATA: towards a database of magnetic structures. I.The commensurate case. J. Appl. Cryst. 49, 1750-1776 (2016).

68. Schoop, L. M. et al. Tunable weyl and dirac states in the nonsymmorphic compound CeSbTe. Sci. Adv. 4, eaar2317 (2018).

69. Kealhofer, D. A. et al. Basal-plane growth of cadmium arsenide by molecular beam epitaxy. Phys. Rev. Mater. 3, 031201 (2019).

70. Chang, T.-R. et al. Type-ii symmetry-protected topological dirac semimetals. Phys. Rev. Lett. 119, 026404 (2017).

71. Guo, P.-J., Yang, H.-C., Liu, K. \& Lu, Z.-Y. Type-ii dirac semimetals in the YPd $_{2}$ Sn class. Phys. Rev. B 95, 155112 (2017).

72. Moll, P. J. W. et al. Transport evidence for fermi-arc-mediated chirality transfer in the dirac semimetal $\mathrm{Cd}_{3} \mathrm{As}_{2}$. Nature 535, $266 \mathrm{EP}$ (2016).
73. Pretko, M. Generalized electromagnetism of subdimensional particles: a spin liquid story. Phys. Rev. B 96, 035119 (2017).

74. Python tight binding open-source package. http://physics.rutgers.edu/pythtb/.

75. Kresse, G. \& Joubert, D. From ultrasoft pseudopotentials to the projector augmented-wave method. Phys. Rev. B 59, 1758-1775 (1999).

76. Kresse, G. \& Furthmüller, J. Efficiency of ab-initio total energy calculations for metals and semiconductors using a plane-wave basis set. Comput. Mater. Sci. 6, 15-50 (1996).

77. Kresse, G. \& Furthmüller, J. Efficient iterative schemes for ab initio total-energy calculations using a plane-wave basis set. Phys. Rev. B 54, 11169-11186 (1996).

78. Inorganic Crystal Structure Database (ICSD) (Fachinformationszentrum Karlsruhe, Karlsruhe, Germany, 2015). Bergerhoff, G. \& Brown, I. D. Crystallographic databases, F. H. Allen et al. (Hrsg.) Chester, International Union of Crystallography, (1987).

79. Ghorashi, S. A. A., Hu, X., Hughes, T. L. \& Rossi, E. Second-order dirac superconductors and magnetic field induced majorana hinge modes. Phys. Rev. B 100, 020509 (2019).

\section{Acknowledgements}

We thank Ady Stern, Ivo Souza, Maia G. Vergniory, Fan Zhang, Chen Fang, and Michael P. Zaletel for helpful discussions. B.J.W., J.C., and B.A.B. acknowledge the hospitality of the Donostia International Physics Center, where parts of this work were carried out. B.J.W. and B.A.B. were supported by the Department of Energy Grant No. DE-SC0016239, the National Science Foundation EAGER Grant No. DMR 1643312, Simons Investigator Grant No. 404513, ONR Grant No. N00014-14-1-0330, the Packard Foundation, the Schmidt Fund for Innovative Research, and a Guggenheim Fellowship from the John Simon Guggenheim Memorial Foundation. Z.W. acknowledges support from the CAS Pioneer Hundred Talents Program. J.C. acknowledges support from the Flatiron Institute, a division of the Simons Foundation. L.M.S. was supported by a MURI grant on TIs from the Army Research Office, Grant No. ARO W911NF-12-1-0461. B.J.W., L.M.S., and B.A.B. were additionally supported by the NSF through the Princeton Center for Complex Materials, a Materials Research Science and Engineering Center DMR-1420541. As discussed in the main text, during the long preparation of this extensive work, simplified toy-models featuring variants of HOFA states were introduced in refs. ${ }^{53,54}$; further comparisons to the results of ref. ${ }^{53}$ are provided in Supplementary Note 11. Corner modes in fragile phases were also recently recognized in refs. ${ }^{32,37}$, and were connected in ref. ${ }^{32}$ to a robust variant of spinless HOFA states distinct from those introduced in this work. Finally, during the preparation of this work, Majorana HOFA states in nodal superconductors were analyzed in ref. ${ }^{79}$.

\section{Author contributions}

All authors contributed equally to the intellectual content of this work. The existence of topological hinge states in layered quadrupole insulating (QI) Dirac semimetals was first recognized by B.A.B. and X.D. in consultation with B.J.W. and J.C. The $s-d$-hybridized spinful QI, related 2D TCI and fragile phases, and layered 3D $\mathcal{T}$-symmetric HOFA Dirac semimetals introduced in this work were proposed by B.J.W. HOFA states in doubly inverted $\mathcal{T}$-symmetric Dirac semimetals were proposed by J.C. Equivalence between the spinless flux-threaded and spinful $s$ - $d$-hybridized formulations of QIs was demonstrated by B.J.W. in consultation with J.C. and B.B. Band representation analyses of the QI obstructed atomic limit and related topological phases were performed by B.J.W., J.C., and B.B. The nested Jackiw-Rebbi formulation of QIs and HOFA states was introduced by B.J.W. under the supervision of B.B. and B.A.B. The list of space groups supporting HOFA Dirac semimetals was obtained by B.J.W., Z.W., J.C., L.M.S., and B.B. Analysis of HOFA states in body-centered semimetals was performed by B.J.W., J.C., and Z.W. Tight-binding, surface, and hinge state calculations were performed by B.J.W. with assistance from J.C. and B.B. Nested Wilson loop calculations were implemented by B.B., J.C., B.J.W., and Z.W. The materials search was performed by B.J.W., Z.W., L.M.S., and X.D. with help from all authors. First-principles and hinge Green's function calculations were performed by Z.W. The manuscript was written by B.J.W. with help from all of the authors. B.A.B. was responsible for the overall research direction.

\section{Competing Interests}

The authors declare no competing interests.

\section{Additional information}

Supplementary information is available for this paper at https://doi.org/10.1038/s41467 020-14443-5.

Correspondence and requests for materials should be addressed to B.J.W., B.B. or B.A.B.

Peer review information Nature Communications thanks the anonymous reviewer(s) for their contribution to the peer review of this work. Peer reviewer reports are available.

Reprints and permission information is available at http://www.nature.com/reprints

Publisher's note Springer Nature remains neutral with regard to jurisdictional claims in published maps and institutional affiliations. 
(c) (i) Open Access This article is licensed under a Creative Commons Attribution 4.0 International License, which permits use, sharing, adaptation, distribution and reproduction in any medium or format, as long as you give appropriate credit to the original author(s) and the source, provide a link to the Creative Commons license, and indicate if changes were made. The images or other third party material in this article are included in the article's Creative Commons license, unless indicated otherwise in a credit line to the material. If material is not included in the article's Creative Commons license and your intended use is not permitted by statutory regulation or exceeds the permitted use, you will need to obtain permission directly from the copyright holder. To view a copy of this license, visit http://creativecommons.org/ licenses/by/4.0/.

(C) The Author(s) 2020 